\title{
Clues and criteria for designing Kitaev spin liquid revealed by thermal and spin excitations of honeycomb iridates $\mathrm{Na}_{2} \mathrm{IrO}_{3}$
}

\author{
Youhei Yamaji ${ }^{1}$, Takafumi Suzuki ${ }^{2}$, Takuto Yamada ${ }^{2}$, Sei-ichiro Suga $^{2}$, Naoki Kawashima ${ }^{3}$, and Masatoshi Imada ${ }^{4}$ \\ ${ }^{1}$ Quantum-Phase Electronics Center, The University of Tokyo, Tokyo, 113-8656, Japan \\ ${ }^{2}$ Graduate School of Engineering, University of Hyogo, Hyogo, Himeji, 670-2280, Japan \\ ${ }^{3}$ Institute for Solid State Physics, The University of Tokyo, Chiba, 277-8581, Japan and \\ ${ }^{4}$ Department of Applied Physics, The University of Tokyo, Tokyo, 113-8656, Japan
}

(Dated: January 22, 2016)

\begin{abstract}
Contrary to the original expectation, $\mathrm{Na}_{2} \mathrm{IrO}_{3}$ is not a Kitaev's quantum spin liquid (QSL) but shows a zig-zag-type antiferromagnetic order in experiments. Here we propose experimental clues and criteria to measure how a material in hand is close to the Kitaev's QSL state. For this purpose, we systematically study thermal and spin excitations of a generalized Kitaev-Heisenberg model studied by Chaloupka et al. in Phys. Rev. Lett. 110, 097204 (2013) and an effective ab initio Hamiltonian for $\mathrm{Na}_{2} \mathrm{IrO}_{3}$ proposed by Yamaji et al. in Phys. Rev. Lett. 113, 107201 (2014), by employing a numerical diagonalization method. We reveal that closeness to the Kitaev's QSL is characterized by the following properties, besides trivial criteria such as reduction of magnetic ordered moments and Néel temperatures: (1) Two peaks in the temperature dependence of specific heat at $T_{\ell}$ and $T_{h}$ caused by the fractionalization of spin to two types of Majorana fermions. (2) In between the double peak, prominent plateau or shoulder pinned at $\frac{R}{2} \ln 2$ in the temperature dependence of entropy, where $R$ is the gas constant. (3) Failure of the linear spin wave approximation at the low-lying excitations of dynamical structure factors. (4) Small ratio $T_{\ell} / T_{h}$ close to or less than 0.03. According to the proposed criteria, $\mathrm{Na}_{2} \mathrm{IrO}_{3}$ is categorized to a compound close to the Kitaev's QSL, and is proven to be a promising candidate for the realization of the QSL if the relevant material parameters can further be tuned by making thin film of $\mathrm{Na}_{2} \mathrm{IrO}_{3}$ on various substrates or applying axial pressure perpendicular to the honeycomb networks of iridium ions. Applications of these characterization to $\left(\mathrm{Na}_{1-x} \mathrm{Li}_{x}\right)_{2} \mathrm{IrO}_{3}$ and other related materials are also discussed.
\end{abstract}

PACS numbers: 75.10.Jm, 75.10.Kt, 75.40.Gb, 75.40.-s

\section{INTRODUCTION}

Enormous efforts to realize quantum spin liquids (QSLs) have been made since the pioneering work of Anderson and Fazekas 1.2 . Geometrically frustrated interactions, for instance antiferromagnetic Heisenberg interactions on a variance of triangular lattice, have been studied as one promising way to realize the QSL state. Recently, the Kitaev model on a honeycomb structure ${ }^{3}$ has attracted attention, because the ground state is exactly proven to be in a QSL phase. In the Kitaev model, two spins on the nearest neighbor sites $i$, and $j$ interact by the Ising-type interaction, $K_{x} S_{i}^{x} S_{j}^{x}, K_{y} S_{i}^{y} S_{j}^{y}$, and $K_{z} S_{i}^{z} S_{j}^{z}$, where the Ising anisotropy axis depends on the three different bonding directions that compose the honeycomb structure. These anisotropic interactions cause a strong frustrated effect, distinct from the typical geometrical frustration.

The QSL state in the Kitaev model contains two distinct phases, namely QSLs with gapless excitations from the ground state and those with only gapful excitations ${ }^{3}$. The gapless QSL appears, when the system is located around the symmetric point where the bond-depending interactions have the same magnitude, $K_{x}=K_{y}=K_{z}$. If one coupling constant becomes much larger than the other ones, for example $\left|K_{z}\right| \gg\left|K_{x}\right|=\left|K_{y}\right|$, the gapped QSL state is stabilized. Interestingly, the both QSL states, regardless of whether the excitation is gapped or gapless, can be described by noninteracting Majo- rana fermions propagating in the background of static $Z_{2}$ gauge fields ( 0 or $\pi$ flux) that are also written by the localized Majorana fermions $\stackrel{4}{*}$. Reflecting the fractionalization of quantum spins into the Majorna fermions, the spin correlations for further neighbor sites become exactly zero in the Kitaev's QSL states, while those for the nearest neighbor survive.

In addition to the ground state properties, low-energy excitations $\underline{\underline{4}}$ and dynamics ${ }^{5}$ of both gapped/gapless QSL states have also been investigated by analytical methods. The ground state is in the 0 flux sector and the lowest excitation by a spin flip from the Kitaev's QSL state is expressed by adding a 'localized' $\pi$-flux pair accompanying an itinerant Majorana fermion. Since the excited $\pi$-flux pair does not propagate in the Kitaev model, non dispersive mode appears in the low-lying excitation, where the excitation energy is nonzero. Such a gapped excitation is confirmed in the dynamical spin structure factor (DSF) that can be observed in inelastic-neutron-scattering experiments. In the gapped QSL phase, a $\delta$-function peak emerges at the lowest excitation energy of the DSF, while a sharp peak with a long tail generating gapless excitations derived from the incoherent part is observed in the gapless QSL phase.

Quite recently, thermal properties for the Kitaev model on the honeycomb structure have been investigated by the quantum Monte Carlo calculations ${ }^{6}$. The Kitaev model always exhibits a two-peak structure in the specific heat, associated with the fractionalization of a single 
quantum spin into two types of Majorana fermions; one is the itinerant (dispersive) Majorana fermion and the other is the localized (dispersionless) Majorana fermion. The two peaks represent the two crossover temperatures associated with the growth of short-range spin correlations (or thermal excitations of the itinerant Majorana fermions) around the high temperature peak and freezing of flux (or the thermal excitation of the localized Majorana fermions) around the low-temperature peak, respectively. The above characteristic features and pictures for the Kitaev's QSL states are expected to be robust against small perturbations such as magnetic fields and Heisenberg-type interactions. However, details of the stability have not been well understood yet for real materials.

In the search for realization of the Kitaev's QSL states, $\mathrm{Na}_{2} \mathrm{IrO}_{3}$ has attracted attention as one candidate material. In $\mathrm{Na}_{2} \mathrm{IrO}_{3}, \mathrm{Ir}^{4+}$ ion can be expressed as a pseudospin with the total angular momentum one-half 7 . We call this pseudospin just as 'spin' hereafter. $\mathrm{IrO}_{6}$ octahedrons in $\mathrm{Na}_{2} \mathrm{IrO}_{3}$ are built into a planar structure parallel to the $a b$ plane and $\operatorname{Ir}^{4+}$ ions constitute a honeycomb structure ${ }^{7,8}$. Furthermore, the neighboring $\mathrm{IrO}_{6}$ octahedrons are connected by sharing two oxygen atoms on an edge. The two oxygen atoms make bridges connecting the neighboring Ir atoms and both Ir-O-Ir bond angles are nearly equal to $90^{\circ}$. Because of the Ir-O-Ir bonds, the perturbative process generates three different anisotropic interactions between $\operatorname{Ir}^{4+}$ ions depending on the Ir-Ir bond directions. In addition, $\mathrm{Ir}^{4+}$ ions interact via direct overlap of their orbitals, generating the Heisenberg-type interaction as well. Thus, both of the Kitaev-type and Heisenberg-type interactions emerge between the neighboring $\operatorname{Ir}^{4+}$ ions 9 , leading to the KitaevHeisenberg model.

Contrary to the initial predictions ${ }^{7,8}, \mathrm{Na}_{2} \mathrm{IrO}_{3}$ undergoes a magnetic phase transition to a zigzag antiferromagnetic order at $T_{N} \sim 15 \mathrm{~K}^{10,11}$. In order to understand the zigzag ordering, several effective models have been proposed and examined so far ${ }^{9,10,12-15}$. Some of them have succeeded in explaining the thermodynamic quantities such as the specific heat and/or the magnetic susceptibility $9,12,14$.

Several $a b$ initio derivations of effective spin Hamiltonians for $\mathrm{Na}_{2} \mathrm{IrO}_{3}$ have shown that low-energy physics of $\mathrm{Na}_{2} \mathrm{IrO}_{3}$ is roughly described by dominant Kitaev's Ising-type exchange interactions, $K_{x}=K_{y} \gtrsim K_{z} \simeq-30$ meV $\stackrel{14}{ }$, while other much smaller interactions including the Heisenberg exchange eventually hold the key for driving the zigzag order. Therefore, one intriguing challenge is to figure out a guideline for materials design that enables the Kitaev's QSL, against the small interactions through control of them by starting with $\mathrm{Na}_{2} \mathrm{IrO}_{3}$.

In this paper, we first report the characterization of $\mathrm{Na}_{2} \mathrm{IrO}_{3}$ based on our numerical studies. Focusing on $a b$ initio effective Hamiltonians for $\mathrm{Na}_{2} \mathrm{IrO}_{3}$, we evaluate how close $\mathrm{Na}_{2} \mathrm{IrO}_{3}$ is located from the Kitaev's QSL phase by introducing several criteria. For the effective
Hamiltonian of $\mathrm{Na}_{2} \mathrm{IrO}_{3}$, we employ a simple generalized Kitaev-Heisenberg Hamiltonian proposed by Chaloupka, Jackeli, and Khaliullin, in Ref. 9 and an ab initio effective Hamiltonian proposed in Ref. 14. In the previous works ${ }^{14.16}$, we discussed the accuracy of these effective models and concluded that the $a b$ initio model proposed in Ref. 14 explains not only thermal properties but also dynamics of this compound.

By comparing the temperature dependences of specific heat $C$ and the equal-time spin correlations as well as the dispersion of the spin excitation based on the linear spin wave approximation and the dynamical spin structure factors $S(\boldsymbol{Q}, \omega)$, we identify three distinct characteristic regions in the phase diagram of the effective Hamiltonians: In addition to the spin liquid phase, the magnetically ordered phase is classified to two distinct regions. Within the magnetically ordered phases of the generalized Kitaev-Heisenberg model, the system is classified to the category I, when the quantum spin system shows a single peak structure in the temperature dependence of $C$. If a quantum spin system shows a two-peak structure in $C$ despite its magnetic order, the system is classified to the category II. When the ground state is the Kitaev's QSL, the system is classified to the third category, namely, the category III. As summarized in Table $C$ for the systems in the category III have two-peak structure commonly to the category II.

As shown later, in the category I, the low-lying excitations of the generalized Kitaev-Heisenberg model in $S(\boldsymbol{Q}, \omega)$, which is induced by flipping a spin, are well described by using the conventional linear spin wave theory, i.e., successfully interpreted as dispersion of (nearly) free magnons. In contrast to the category I, a system categorized as the category II shows that the low-lying excitations in $S(\boldsymbol{Q}, \omega)$ are not captured by the linear spin wave theory on a qualitative level. The breakdown of the linear spin wave theory is a common property in both categories II and III, although the spin wave analysis has been employed in comparing effective Hamiltonians with experimental results of $A_{2} \operatorname{IrO} 3(A=\mathrm{Na}, \mathrm{Li})^{17,18}$ and another Kitaev's QSL candidate $\alpha-\mathrm{RuCl}_{3} 19$. The above different categories are not necessarily separated by the phase transition: Categories I and II are separated from III by a quantum phase transition, while the states in the categories I and II can be connected smoothly. All of highly generalized Kitaev models treated in this paper can be represented by one of these three empirical categories consistently in all the physical quantities studied. Thus, the spin excitation spectra would provide us with useful supplementary data for the classification.

We then examine the nature of the ground state of the $a b$ initio Hamiltonian of $\mathrm{Na}_{2} \mathrm{IrO}_{3}$ in light of our proposed categorization. We show that the $a b$ initio Hamiltonian of $\mathrm{Na}_{2} \mathrm{IrO}_{3}$ belongs to the category II. This supports that a better chance of material design to realize the Kitaev's QSL may exist through a realistic tuning of the material parameters of $\mathrm{Na}_{2} \mathrm{IrO}_{3}$. In this context, we propose that the temperature dependence of the entropy as well 
as the energy scales measured by the ratio of the higherand lower-temperature peaks in $C$ give a quantitative measure of the closeness to the Kitaev's QSLs in the category II. We believe that this proposal offers a guideline for the materials design.

\section{MODEL AND METHOD}

\section{A. Effective Hamiltonian}

In this paper, we examine magnetic and thermal excitations of the generalized Kitaev-Heisenberg model proposed in Ref. 9 and the ab initio Hamiltonian of $\mathrm{Na}_{2} \mathrm{IrO}_{3}$ proposed in Ref. 14 .

\section{Generalized Kitaev-Heisenberg model}

The generalized Kitaev-Heisenberg model is one of the simplest models that describe both Kitaev's QSL and zigzag magnetic orders. The model is parameterized by two exchange couplings, namely the Kitaev-type coupling $K=2 A \sin \varphi$ and the Heisenberg coupling $J=A \cos \varphi$, as

$$
\hat{H}_{\mathrm{CJK}}=\sum_{\Gamma=X, Y, Z} \sum_{\langle i, j\rangle \in \Gamma} \overrightarrow{\hat{S}}_{i}^{T} \mathcal{J}_{\Gamma} \overrightarrow{\hat{S}}_{j}
$$

where $A$ has the dimension of energy and $\overrightarrow{\hat{S}}_{i}$ is an $\mathrm{SU}(2)$ spin operators $\left(\hat{S}_{i}^{x}, \hat{S}_{i}^{y}, \hat{S}_{i}^{z}\right)$ at the $i$-th site, and the matrices of the exchange couplings for the three nearestneighbor bonds, $X$-, $Y$-, and $Z$-bond (see Fig. 1(a)), are defined as

$$
\begin{gathered}
\mathcal{J}_{X}=\left[\begin{array}{ccc}
K+J & 0 & 0 \\
0 & J & 0 \\
0 & 0 & J
\end{array}\right], \\
\mathcal{J}_{Y}=\left[\begin{array}{ccc}
J & 0 & 0 \\
0 & K+J & 0 \\
0 & 0 & J
\end{array}\right],
\end{gathered}
$$

and

$$
\mathcal{J}_{Z}=\left[\begin{array}{ccc}
J & 0 & 0 \\
0 & J & 0 \\
0 & 0 & K+J
\end{array}\right]
$$

The ground states of the generalized KitaevHeisenberg model range from the Kitaev's QSLs to trivial magnetically ordered states depending on the control parameter $\varphi$ for fixed $A$ taken positive. For $\varphi \sim 90^{\circ}$ and $\varphi \sim 270^{\circ}$, the Kitaev's QSLs appear. When the system size $N=24$ is considered, the stripy, Néel, zigzag, and ferromagnetic orders appear for $-76,1^{\circ} \lesssim \varphi \lesssim-33.8^{\circ}$, $-33.8^{\circ} \lesssim \varphi \lesssim 87.7^{\circ}, 92.2^{\circ} \lesssim \varphi \lesssim 161.8^{\circ}$, and $161.8^{\circ} \lesssim$ $\varphi \lesssim 251.8^{\circ}$, respectively, determined in Ref. 9 by exact diagonalization with $N=24$.

\section{Ab initio Hamiltonian of $\mathrm{Na}_{2} \mathrm{IrO}_{3}$}

Let us consider a highly generalized form of the KitaevHeisenberg model on the honeycomb structure for the purpose of bridging to the realistic and ab initio Hamiltonian. The Hamiltonian is given as

$$
\hat{H}_{\lambda}=\sum_{\Gamma=X, Y, Z, Z_{2 \mathrm{nd}}, C_{3 \mathrm{rd}}} \sum_{\langle i, j\rangle \in \Gamma} \overrightarrow{\hat{S}}_{i}^{T} \mathcal{J}_{\Gamma}(\lambda) \overrightarrow{\hat{S}}_{j},
$$

where the matrices of the exchange couplings for the three different nearest-neighbor $(X-, Y$-, and $Z$-bonds), the second neighbor $\left(Z_{2 \mathrm{nd}}\right.$-bond $)$, and the third neighbor $\left(C_{3 \mathrm{rd}}\right.$-bond $)$ bonds are given as

$$
\begin{gathered}
\mathcal{J}_{X}(\lambda)=\left[\begin{array}{ccc}
K^{\prime} & 0 & 0 \\
0 & 0 & 0 \\
0 & 0 & 0
\end{array}\right]+\lambda\left[\begin{array}{ccc}
0 & I_{2}^{\prime \prime} & I_{2}^{\prime} \\
I_{2}^{\prime \prime} & J^{\prime \prime} & I_{1}^{\prime} \\
I_{2}^{\prime} & I_{1}^{\prime} & J^{\prime}
\end{array}\right], \\
\mathcal{J}_{Y}(\lambda)=\left[\begin{array}{ccc}
0 & 0 & 0 \\
0 & K^{\prime} & 0 \\
0 & 0 & 0
\end{array}\right]+\lambda\left[\begin{array}{ccc}
J^{\prime \prime} & I_{2}^{\prime \prime} & I_{1}^{\prime} \\
I_{2}^{\prime \prime} & 0 & I_{2}^{\prime} \\
I_{1}^{\prime} & I_{2}^{\prime} & J^{\prime}
\end{array}\right], \\
\mathcal{J}_{Z}(\lambda)=\left[\begin{array}{ccc}
0 & 0 & 0 \\
0 & 0 & 0 \\
0 & 0 & K
\end{array}\right]+\lambda\left[\begin{array}{ccc}
J & I_{1} & I_{2} \\
I_{1} & J & I_{2} \\
I_{2} & I_{2} & 0
\end{array}\right], \\
\mathcal{J}_{Z_{2 \mathrm{nd}}}(\lambda)=\lambda\left[\begin{array}{ccc}
J^{(2 \mathrm{nd})} & I_{1}^{(2 \mathrm{nd})} & I_{2}^{(2 \mathrm{nd})} \\
I_{1}^{(2 \mathrm{nd})} & J^{(2 \mathrm{nd})} & I_{2}^{(2 \mathrm{nd})} \\
I_{2}^{(2 \mathrm{nd})} & I_{2}^{(2 \mathrm{nd})} & K^{(2 \mathrm{nd})}
\end{array}\right], \\
\mathcal{J}_{C_{3 \mathrm{rd}}}(\lambda)=\lambda\left[\begin{array}{ccc}
J^{(3 \mathrm{rd})} & 0 & 0 \\
0 & J^{(3 \mathrm{rd})} & 0 \\
0 & 0 & J^{(3 \mathrm{rd})}
\end{array}\right],
\end{gathered}
$$

respectively. The details of the bond are illustrated in Fig. 1 (a). Here a parameter $\lambda$ is introduced to interpolate the $a b$ initio Hamiltonian for $\mathrm{Na}_{2} \mathrm{IrO}_{3}$ at $\lambda=1$ and the Kitaev model at $\lambda=0$. The $a b$ initio estimates of the exchange couplings are summarized in Table II. This model at $\lambda=1$ well explains not only the thermal properties, such as the specific heat for $5 \mathrm{~K}<T<40 \mathrm{~K}$ and the susceptibility for $5 \mathrm{~K}<T<400 \mathrm{~K}$, but also the low-lying magnetic excitations ${ }^{14,16}$.

While the ground state of the interpolated Hamiltonian is in the gapless QSL phase at $\lambda=0$, the zigzag-type antiferromagnetic order is stabilized at $\lambda=1 \stackrel{14}{ }$. Then, a quantum phase transition from the topological QSL state to the magnetic ordered state has to occur at least once, between $\lambda=0$ and 1 .

\section{B. Specific heat}

The specific heat of the spin Hamiltonians, $\hat{H}_{\mathrm{CJK}}$ and $\hat{H}_{\lambda}$, is calculated by using exact energy spectra up to 
TABLE I: Three categories characterized by thermal and magnetic excitations; peak structures in the temperature dependences of the specific heat $C$ and nature of quasiparticles (QP). The nature of QP can be discussed from the comparison between dynamical spin structure factors $S(\boldsymbol{Q}, \omega)$ and linear spin wave theory (SW).

\begin{tabular}{cllll}
\hline \hline & $C$ & LRO & QP & $S(\boldsymbol{Q}, \omega)$ vs. SW \\
\hline I. & single peak & magnetic & free magnon & consistent \\
II. & two peaks & magnetic & correlated magnon & discrepant \\
III. & two peaks & no & Majorana & inconsistent \\
\hline \hline
\end{tabular}

(a)

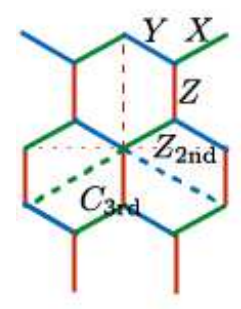

(b)

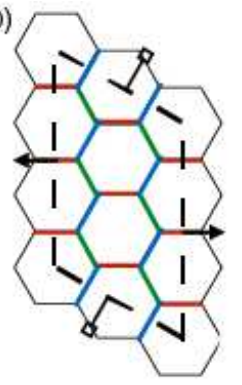

(c)

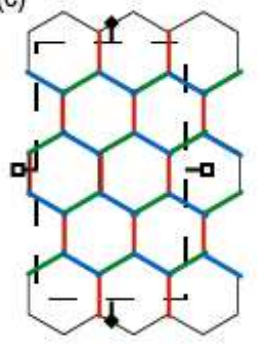

(d)

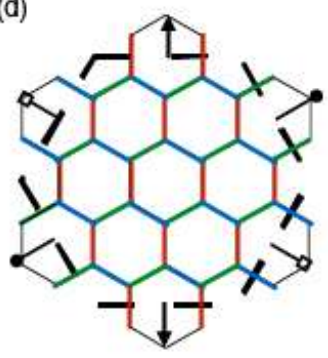

(e)

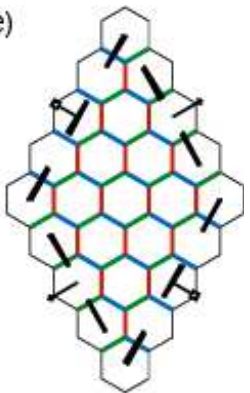

FIG. 1: (Color online) (a) Honeycomb structure model. Red, blue, and green lines denote $Z$-, $Y$-, and $X$ - bond, respectively. Dotted (dashed) lines represent the second (third) neighbor bonds. For the second neighbor bonds, only the bond perpendicular to $Z$-bond, namely, $Z_{2 \text { nd }}$-bond is shown because the amplitude for the second neighbor interactions in the $a b$ initio Hamiltonian is quite small and can be ignored ${ }^{14}$. Here, $C_{3 \mathrm{rd}}$ denotes the set of the third neighbor bonds. (b-e) $N=12,16,24$, and 32 -site clusters, respectively.

TABLE II: Exchange couplings of the $a b$ initio effective Hamiltonian for $\mathrm{Na}_{2} \mathrm{IrO}_{3}$ derived in Ref. 14 .

\begin{tabular}{ccccccc}
\hline \hline $\mathcal{J}_{Z}(\mathrm{meV})$ & $K$ & $J$ & $I_{1}$ & $I_{2}$ & & \\
& -30.7 & 4.4 & -0.4 & 1.1 & & \\
\hline $\mathcal{J}_{X, Y}(\mathrm{meV})$ & $K^{\prime}$ & $J^{\prime}$ & $J^{\prime \prime}$ & $I_{1}^{\prime}$ & $I_{2}^{\prime}$ & $I_{2}^{\prime \prime}$ \\
& -23.9 & 2.0 & 3.2 & 1.8 & -8.4 & -3.1 \\
\hline $\mathcal{J}_{Z_{2 \mathrm{nd}}}(\mathrm{meV})$ & $K^{(2 \mathrm{nd})}$ & $J^{(2 \mathrm{nd})}$ & $I_{1}^{(2 \mathrm{nd})}$ & $I_{2}^{(2 \mathrm{nd})}$ & & \\
& -1.2 & -0.8 & 1.0 & -1.4 & & \\
\hline $\mathcal{J}_{C_{3 \mathrm{rd}}}(\mathrm{meV})$ & $J^{(3 \mathrm{rd})}$ & & & & & \\
& 1.7 & & & & & \\
\hline \hline
\end{tabular}

$N=16$ sites, and is estimated by employing thermal pure quantum states ${ }^{20,21}$ for the 24 - and 32 -site clusters with the periodic boundary condition. The finite size clusters used in the following are illustrated in Fig:1(b)(e).

Here we briefly summarize the construction of thermal pure quantum (TPQ) states following Ref. 20. A TPQ state at infinite temperatures is simply given by a random vector,

$$
\left|\phi_{+\infty}\right\rangle=\sum_{i=0}^{2^{N}-1} c_{i}|i\rangle,
$$

where $|i\rangle$ is represented by the real-space $S=1 / 2$ basis and specified by a binary representation of decimal and $\left\{c_{i}\right\}$ is a set of random complex numbers with the normalization condition $\sum_{i=0}^{2^{N}-1}\left|c_{i}\right|^{2}=1$. Then, by utilizing the Lanczos steps with a Hamiltonian $\hat{H}$, the TPQ states at lower temperatures are constructed as follows: Starting with an initial vector $\left|\Phi_{0}\right\rangle=\left|\phi_{+\infty}\right\rangle$, the $k$-th step Lanczos vector $\left|\Phi_{k}\right\rangle(k \geq 1)$ is constructed as

$$
\left|\Phi_{k}\right\rangle=\frac{\hat{H}\left|\Phi_{k-1}\right\rangle}{\sqrt{\left\langle\Phi_{k-1}\left|\hat{H}^{2}\right| \Phi_{k-1}\right\rangle}} .
$$

The above $k$-th step Lanczos vector is a TPQ state at a finite temperature $T$. The corresponding inverse temperature $\beta=\left(k_{B} T\right)^{-1}$ is determined through the following formula 20 ,

$$
\beta=\frac{2 k_{B} k}{\Lambda-\left\langle\Phi_{k}|\hat{H}| \Phi_{k}\right\rangle}+O(1 / N)
$$

where $k_{B}$ is the Boltzmann constant and $\Lambda$ is a constant larger than maxima of $\langle\hat{H}\rangle$. In other word, a TPQ state at $T$ is given as,

$$
\left|\phi_{T}\right\rangle=\left|\Phi_{k}\right\rangle .
$$

The specific heat and entropy of $\hat{H}$ are then estimated by using TPQ states $\left|\phi_{T}\right\rangle$. The thermodynamics and statistical mechanics tell us several prescriptions to calculate the specific heat and the entropy. Here, we calculate the specific heat $C$ by using the derivative of internal energy with respect to the temperature as

$$
C=\frac{d\left\langle\phi_{T}|\hat{H}| \phi_{T}\right\rangle}{d T}
$$


which is empirically known to be intruded by less statistical errors in comparison with results obtained through thermal fluctuations of $\hat{H}$. In the present paper, the entropy $S$ is estimated by integrating $C / T$ from high temperatures as

$$
S=N k_{B} \ln 2-\int_{T}^{+\infty} d T^{\prime} \frac{C}{T^{\prime}},
$$

where $C \propto T^{-2}$ is assumed in the above integral for the high temperature asymptotic behavior of $C$. Here, we note that, for the specific heat and entropy defined in Eq.(15) and Eq.(16), respectively, of the lattice models, it is convenient to use $N k_{B}$, instead of the gas constant $R$ used in experiments.

\section{Equal-time spin correlation}

In comparison with the peak structures of the specific heat, we examine temperature dependence of the equaltime spin correlations. For short-range spin correlations, we calculate expectation values of spin operators $\hat{S}_{i}^{\mu} \hat{S}_{j}^{\mu}$ at a finite temperature $T$ with the thermal pure quantum $\operatorname{states}^{20,21}\left|\phi_{T}\right\rangle$ as

$$
\left\langle\hat{S}_{i}^{\mu} \hat{S}_{j}^{\mu}\right\rangle_{T} \equiv\left\langle\phi_{T}\left|\hat{S}_{i}^{\mu} \hat{S}_{j}^{\mu}\right| \phi_{T}\right\rangle
$$

for the nearest-neighbor pairs $\langle i, j\rangle$. Long-range spin correlations are characterized by the peak value in the momentum dependence of the equal-time spin structure factor $S_{T}(\boldsymbol{q})$ defined by Fourier transformation of $\left\langle\hat{S}_{i}^{\mu} \hat{S}_{j}^{\mu}\right\rangle_{T}$,
as

$$
S_{T}(\boldsymbol{q})=\frac{1}{N} \sum_{\mu=x, y, z} \sum_{\ell=0}^{N-1}\left\langle\hat{S}_{0}^{\mu} \hat{S}_{\ell}^{\mu}\right\rangle_{T} \cos \left(\boldsymbol{q} \cdot \boldsymbol{R}_{\ell}\right),
$$

at $\boldsymbol{q}=Q$ where $\boldsymbol{R}_{\ell}$ is the position vector of the $\ell$-th site and $\boldsymbol{Q}$ is the momentum at the maximum.

\section{Dynamical spin structure factors}

To discuss magnetic excitations by a spin flip, we focus on the dynamical spin structure factor (DSF) at zero temperature. The DSF is defined as

$$
S^{\mu \nu}(\boldsymbol{Q}, \omega) \equiv-\frac{1}{\pi} \lim _{\epsilon \rightarrow+0} \operatorname{Im}\left\langle\phi_{0}\left|\hat{S}_{\boldsymbol{Q}}^{\mu \dagger} \frac{1}{\omega+E_{0}+i \epsilon-\mathcal{H}} \hat{S}_{\boldsymbol{Q}}^{\nu}\right| \phi_{0}\right\rangle
$$

where $\phi_{0}$ is the ground state of $\mathcal{H}$ with the ground state energy $E_{0}$. The spin operator $\hat{S}_{\boldsymbol{Q}}^{\mu}$ is the Fourier transform of $\hat{S}_{i}^{\mu}$, where $\mu, \nu$ stand for $x, y$ or $z$ component. After calculating $\phi_{0}$ and $E_{0}$ by the Lanczos method, $S^{\mu \nu}(\boldsymbol{Q}, \omega)$ is obtained by the continued fraction expansion ${ }^{22,23}$.
In this paper, we focus on the sum of diagonal elements, namely $S(\boldsymbol{Q}, \omega)=\sum_{\mu=x, y, z} S^{\mu \mu}(\boldsymbol{Q}, \omega)$. In the generalized Kitaev-Heisenberg model, the symmetry of the model Hamiltonian ensures that the off-diagonal component of the DSF becomes exactly zero. However, in general, $S^{\mu \nu}(\boldsymbol{Q}, \omega)$ may have non-zero off-diagonal elements, if the off-diagonal elements of $\hat{\mathcal{J}}_{\Gamma_{p}}^{\mu \nu}$ are non-zero. The contribution from such off-diagonal element is proportional to the Fourier transform of the corresponding time-displaced spin correlation, such as $\left\langle\hat{S}_{i}^{x}(t) \hat{S}_{j}^{y}(0)\right\rangle$ and $\left\langle\hat{S}_{i}^{x}(t) \hat{S}_{j}^{z}(0)\right\rangle$. Since the amplitude of the spin correlation is scaled by the amplitude of the matrix element of $\hat{\mathcal{J}}_{\Gamma_{p}}^{\mu \nu}$, the diagonal elements are dominant. The diagonal elements of the $a b$ initio Hamiltonian is indeed dominant over the off-diagonal elements, and $S(\boldsymbol{Q}, \omega)$ is expected to contain the main contribution of the spin excitations.

\section{THERMAL AND SPIN EXCITATIONS}

\section{A. Results of generalized Kitaev-Heisenberg model}

To gain insights into the nature and proximity of the Kitaev's QSL, we compare the specific heat, the linear spin wave dispersion, and the dynamical spin structure factor (DSF), which allows a classification of the spin Hamiltonians $\hat{H}_{\mathrm{CJK}}$ to three distinct categories as summarized in Table [I For several choices of the parameter $\varphi$ for the generalized Kitaev-Heisenberg model, we classify the nature of the spin and thermal excitations. The resultant categorization is summarized in Table III.

The specific heat $C$ for the generalized KitaevHeisenberg model is shown in Fig. 2. Here, we show the results for $N=12,16,24$, and 32. Except the Kitaev's QSLs at $\varphi=90^{\circ}$, we see no strong $N$ dependence for the second-largest and largest system sizes $N=24$ and 32. For the trivially ordered states at $\varphi=0^{\circ}$ and $180^{\circ}$, the temperature dependences of $C$ shows a Schottky-like single peak within the error bars. In the thermodynamic limit, the peak may evolve into the anomaly (divergence or peak) expected by the growth of spin correlations accompanied by the transition to the long-range order. In contrast, for the Kitaev's QSL states at $\varphi=90^{\circ}$ ( and equivalently at $\varphi=270^{\circ}$ ), there are two peaks in the temperature dependences of $C$, which is a hallmark of thermal fractionalization proposed in Ref. 6, as is introduced in Sec. I The low-temperature peak of $C$ may be associated with the contribution from the thermal flux excitations (or the thermal excitations of the localized Majorana fermions), while the high-temperature peak may represent the excitation of the itinerant Majorana fermions. The category II represented by $\varphi=100^{\circ}$ and $240^{\circ}$ is the same as the category I as to the presence of the magnetic order, while the two-peak structure of $C$ exists similarly to the category III. The ordered state 


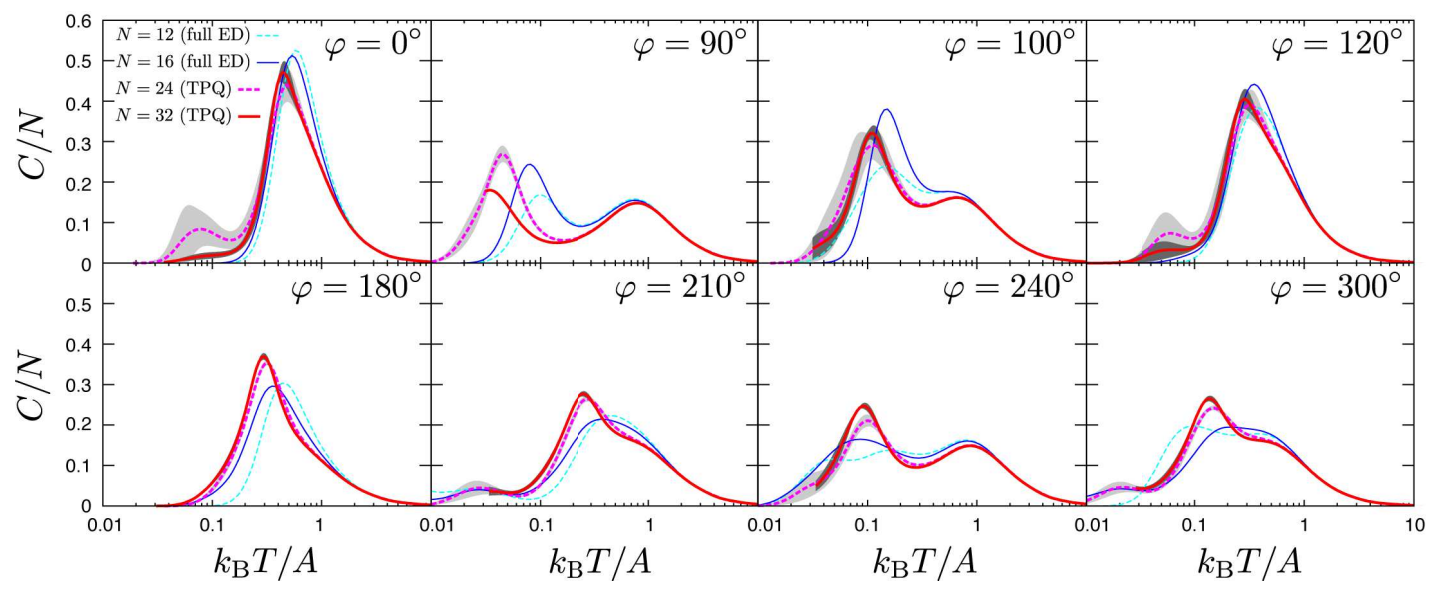

FIG. 2: (Color online) Temperature dependences of the specific heat $C$ of the generalized Kitaev-Heisenberg model $\hat{H}_{\mathrm{CJK}}$. The results for $N=12$ and $N=16$ obtained by fully diagonalizing $\hat{H}_{\mathrm{CJK}}$ (denoted as "full ED") are illustrated with thin broken and thin solid (blue) curves, respectively. For $N=24$ and $N=32$ (thick broken and thick solid (red) curves), the thermal pure quantum (TPQ) states $^{20}$ are employed. The possible errors of TPQ due to the truncation of the Hilbert space are shown in $C$ by shaded (gray) belts, which is estimated by using the standard deviation of the results obtained from 4 to 36 initial random wave functions at the high temperature limit, $k_{B} T / A \rightarrow+\infty$. From the top leftmost to top rightmost panels, $C / N$ is shown for $\varphi=0^{\circ}, 90^{\circ}, 100^{\circ}$, and $120^{\circ}$ in this order. The same quantities are shown for $\varphi=180^{\circ}, 210^{\circ}, 240^{\circ}$, and $300^{\circ}$ in this order from the bottom leftmost to bottom rightmost.

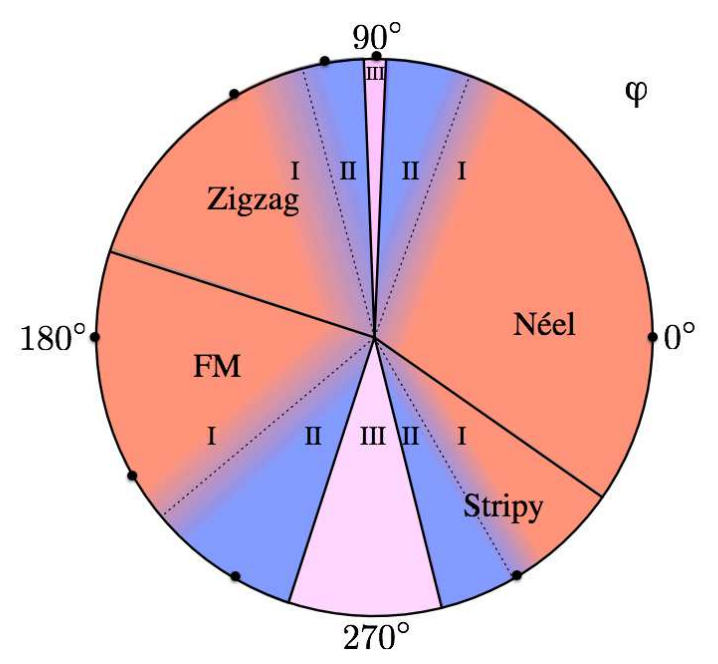

FIG. 3: (Color online) Categorization of ground states of generalized Kitaev-Heisenberg model. Phase boundaries depicted by the solid lines are drawn by using the results in Ref. 9 . Solid dots represent the parameters shown in Figs. 204 and 5 Dashed lines separate whether or not the two-peak structure in the temperature dependence of the specific heat is observed within the magnetic ordered phase, namely the crossover border between the categories I (blue (lightly shaded) area) and II (red (dark shaded) area). Note that the dotted lines do not represent the phase boundary.

at $\varphi=300^{\circ}$ is located almost on the border between the category I and category II. Although the two-peak structure itself is not a unique feature of the Kitaev's
QSL, we will propose later that the entropy at temperatures between the two peaks in $C$ serves as a hallmark of the closeness to the Kitaev's QSL. In Fig. 3, we show the schematic illustration for the categorization obtained from the temperature dependence of $C$ and the presence of the magnetic order.

In Fig. 4 we compare the temperature dependence of the long-range part of the spin correlation represented by the peak in $S_{T}(\boldsymbol{Q})$ and the short-range part represented by $\left\langle\hat{S}_{i}^{\mu} \hat{S}_{j}^{\mu}\right\rangle_{T}$. While, in the category I, the short-range spin correlations $\left\langle\hat{S}_{i}^{\mu} \hat{S}_{j}^{\mu}\right\rangle_{T}$ and the long-range spin correlations $S_{T}(\boldsymbol{Q})$ at the ordering wave vector $\boldsymbol{Q}$ grow simultaneously around the temperature where $C$ has the single peak, $\left\langle\hat{S}_{i}^{\mu} \hat{S}_{j}^{\mu}\right\rangle_{T}$ and $S_{T}(\boldsymbol{Q})$ grow independently in the category II represented by $\varphi=100^{\circ}$ and $240^{\circ}$. The growth of the spin correlation changes from the category I to the category II around $\varphi=300^{\circ}$. In the category II, $\left\langle\hat{S}_{i}^{\mu} \hat{S}_{j}^{\mu}\right\rangle_{T}$ grows as temperature falls to $T_{h}$, which corresponds to the high-temperature peak in $C$, and saturates below $T_{h}$. On the other hand, the long-range spin correlations represented by $S_{T}(\boldsymbol{Q})$ grow significantly around the temperatures $T_{\ell}$ where $C$ has the low-temperature peak, in the category II. In the category III, the shortrange spin correlation grows at $T_{h}$, while the long-range part does not show appreciable temperature dependence even at $T_{\ell}$ in contrast to the category II. The low temperature peak in $C$ in the category III arises from an entirely different mechanism from that in the category II, as we already mentioned. The difference between the category 


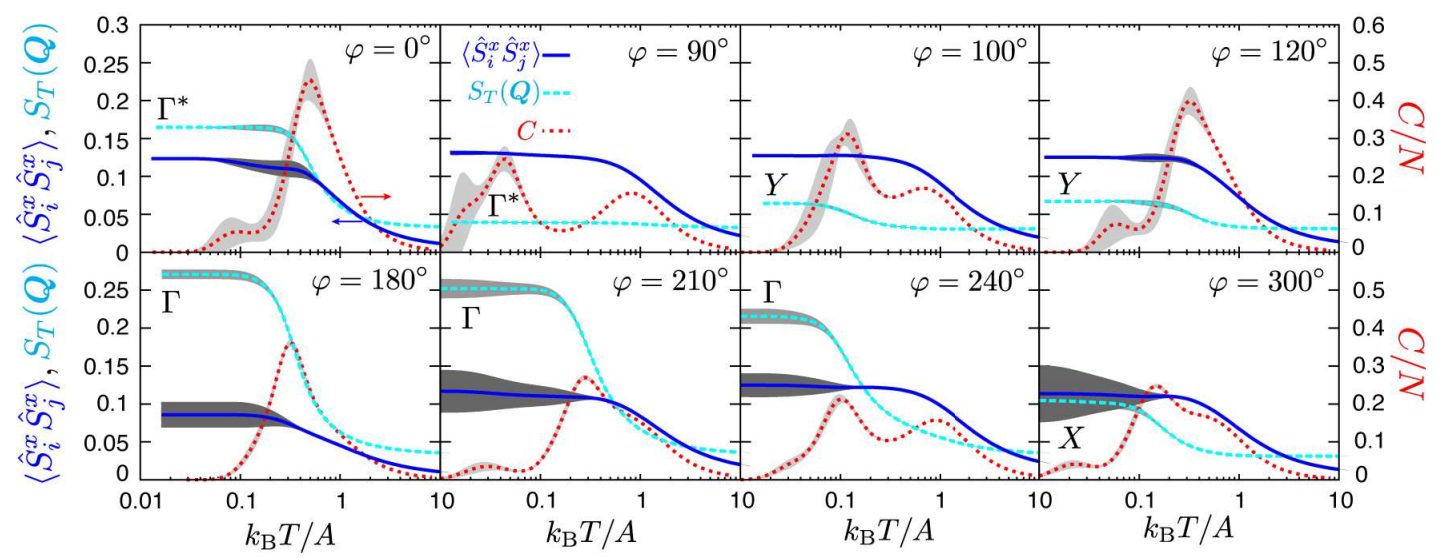

FIG. 4: (Color online) Temperature dependence of short-range spin correlation $\left\langle\hat{S}_{i}^{x} \hat{S}_{j}^{x}\right\rangle_{T}$ for $X$-bond and long-range spin correlation $S_{T}(\boldsymbol{Q})$ in comparison with the specific heat $C$. The results for $N=24$ by employing the thermal pure quantum states $^{20}$ are shown. The possible errors of TPQ due to the truncation of the Hilbert space are shown in $\left\langle\hat{S}_{i}^{x} \hat{S}_{j}^{x}\right\rangle_{T}, S_{T}(\boldsymbol{Q})$, and $C$ by shaded (gray) belts, which is estimated by using the standard deviation of the results obtained from 4 to 36 initial random wave functions at the high temperature limit, $k_{B} T / A \rightarrow+\infty$. For $S_{T}(\boldsymbol{Q})$, following points in Fig. 5 (i) are selected as the wave vector $\boldsymbol{Q}$; the $\Gamma, Y, \Gamma^{*}$, and $X$ points are selected since $S_{T}(\boldsymbol{Q})$ for each value of $\varphi$ has maxima at the momentum. These points represent the Bragg points of the ferromagnetic (FM), zigzag, Néel, and stripy orders, respectively. The momentum $\boldsymbol{Q}$ is consistent with the phase diagram in Fig 3. From the top leftmost to top rightmost panels, the results are shown for $\varphi=0^{\circ}$, $90^{\circ}, 100^{\circ}$, and $120^{\circ}$ in this order. The same quantities are shown for $\varphi=180^{\circ}, 210^{\circ}, 240^{\circ}$, and $300^{\circ}$ in this order from the bottom leftmost to bottom rightmost.

II and the category III is evident in the temperature dependence of the peak of $S_{T}(\boldsymbol{Q})$ in comparison with that of $\left\langle\hat{S}_{i}^{\mu} \hat{S}_{j}^{\mu}\right\rangle_{T}$, as shown in Fig团

Based on the above results, we categorize the quantum phases obtained for the generalized Kitaev-Heisenberg model. The summary is shown in Table III and Fig. 3.

TABLE III: Categorization of ground states for several choices of $\varphi$ of the generalized Kitaev-Heisenberg model. Definition of the categories I - III is shown in Table 1

\begin{tabular}{clc}
\hline \hline$\varphi$ & quantum phase category \\
\hline $0^{\circ}$ & Néel & I. \\
$90^{\circ}$ & Kitaev's QSL & III. \\
$100^{\circ}$ & zigzag & II. \\
$120^{\circ}$ & zigzag & I. \\
$180^{\circ}$ & ferromagnetic & I. \\
$210^{\circ}$ & ferromagnetic & I. \\
$240^{\circ}$ & ferromagnetic & II. \\
$270^{\circ}$ & Kitaev's QSL & III. \\
$300^{\circ}$ & stripy & I/II. \\
\hline \hline
\end{tabular}

By comparing the low-lying excitations of the dynamical spin structure factors (DSFs) with the linear spin wave approximation, we further confirm that the categorization is robust. In Fig [5, we show both results of the DSF $S(\boldsymbol{Q}, \omega)$ for $N=24$ and of the linear spin wave calculations.

We start from the results for the simplest case. At $\varphi=0^{\circ}$ and $180^{\circ}$, the model becomes the antiferro- magnetic/ferromagnetic Heisenberg model. Therefore, the low-lying excitation of the DSF is expected to be well explained by the spin wave mode. At $\varphi=180^{\circ}$, all poles in the DSF are perfectly located on the spin wave mode. In the antiferromagnetic case at $\varphi=0^{\circ}$, the low-lying excitation agrees with the linear spin wave mode by introducing a renormalization factor $a$. $a$ is estimated from the best fitting of spin wave dispersions for the poles of the low-lying excitations in the DSF; $S\left(\boldsymbol{Q}, \omega_{\text {lowest }}\right) \sim a \times \omega_{\mathrm{LSW}}(\boldsymbol{Q})$, where $S\left(\boldsymbol{Q}, \omega_{\text {lowest }}\right)$ denotes the poles of the lowest excitation in the DSF and $\omega_{\mathrm{LSW}}(\boldsymbol{Q})$ is the linear spin wave mode. At $\varphi=0^{\circ}$, we obtain $a \sim 1.3$, which is the upper limit because the positions of poles are affected by the system size. (The size dependence becomes strong especially at the symmetric wave-number points. ) As well studied in Refs. 24, 25, the exact low-lying excitations in the Heisenberg models are described by the linear spin wave mode with an $O(1)$ renormalization factor $a$. The renormalization factor is $a=\frac{\pi}{2}$ in the $\mathrm{S}=1 / 2$ spin chain case $\underline{\underline{24}}$. This value can be an indicator for the renormalization of the quantum fluctuation. As shown below, we observe the two-peak structure in the temperature dependence of the specific heat $C$, when $a \gtrsim 1.5$.

In contrast, magnetic excitations described by the poles in the DSF are completely different from coherent magnetic excitations in the Kitaev's QSL phase at $\varphi=90^{\circ}\left(270^{\circ}\right)$. This is due to diverging quantum fluctuations in the Kitaev's QSL phase and massive degeneracy of classical spin orders spoils the linear spin wave 


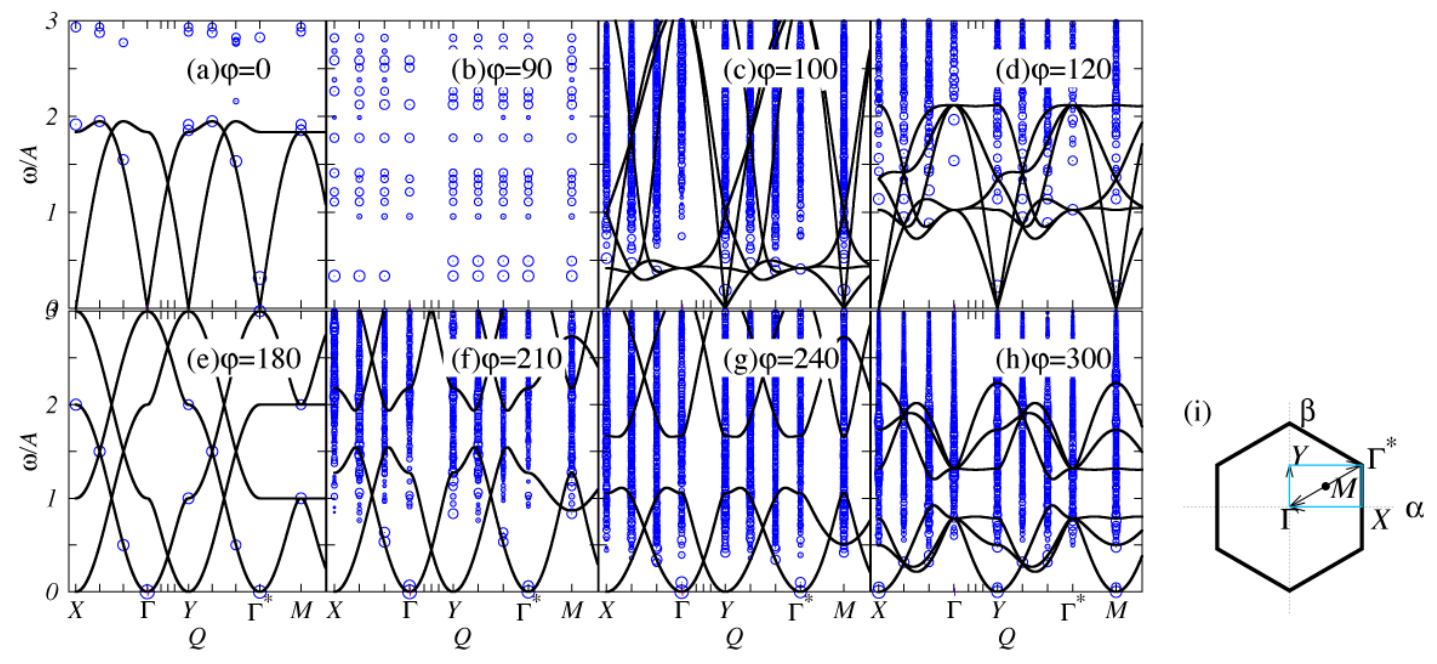

FIG. 5: (Color online) Dynamical spin structure factors of generalized Kitaev-Heisenberg models for 24-site cluster. Area of each circle is proportional to the intensity in logarithmic scale. Abscissa represents labeled points in (i). From the top leftmost to top rightmost panels, $S(\boldsymbol{Q}, \omega)$ is shown for (a) $\varphi=0^{\circ}$, (b) $90^{\circ}$, (c) $100^{\circ}$, and (d) $120^{\circ}$ in this order. $S(\boldsymbol{Q}, \omega)$ is shown for (e) $\varphi=180^{\circ}$, (f) $210^{\circ}$, (g) $240^{\circ}$, and (h) $300^{\circ}$ in the order from the bottom leftmost to bottom rightmost. Here, the results of the linear spin wave theory are shown in solid curves. In order to compare the spin wave dispersions with the low-lying excitations, each spin wave result in the top panels is multiplied by a constant. The multiplication constants for (a), (c) and (d) are 1.3, 1.65, and 1.45, respectively. The spin wave results in the lower panels are drawn without such tuning constant. For the results in the zigzag and stripy phase, (c), (d), and (h), we also plot the spin wave dispersions obtained from the case where the ordered state is rotated by $2 \pi / 3$.

analysis. Below, we explain the low-lying excitations of the DSFs, when the system approaches the Kitaev's QSL phase from the deep inside of the magnetic ordered phase.

First, we focus on the positive Kitaev-coupling case for $0 \leq \varphi \leq 180^{\circ}$. For $0 \leq \varphi \leq 180^{\circ}$ in the magnetic ordered phases, the low-lying excitation of the DSF can be explained by the correlated/renormalized magnon excitation. The fitting parameter $a$ is always larger than the unity; the low-lying excitation becomes hard in comparison with the linear spin wave mode. When the system approaches the Kitaev's QSL phase around $90^{\circ}$, $a$ drastically increases. Though not shown in the figure, we obtain $a \sim 1.85$ at $\varphi=86^{\circ}$. At $\varphi=100^{\circ}$ in the category II, the ground state is the zigzag ordered state. From Fig. 2 we confirm the two-peak structure in the temperature dependence of $C$. The renormalization factor $a$ at $\varphi=100^{\circ}$ is estimated as $a \sim 1.65$ and is larger than that of the $\mathrm{S}=1 / 2$ chain case. When the system goes into the deep inside of the magnetic phase, the factor $a$ decreases and crosses $a \sim 1.5$, where the two-peak structure is almost smeared out in $C$. At $\varphi=120^{\circ}$ in the category $\mathrm{I}, a$ is about 1.45. While the ground state is still in the zigzag ordered phase, the temperature dependence of $C$ shows the usual single peak.

Next, we see the results for the negative Kitaev coupling case for $180^{\circ} \leq \varphi \leq 360^{\circ}$. In contrast to the positive Kitaev coupling case, the low-lying excitation in the DSF can be well explained by free magnon picture for $K<0$ in the magnetic ordered phases: The spin wave mode except the $\mathrm{M} / \Gamma\left(\Gamma^{*}\right)$ point can explain the low-lying excitations of the DSF without the renormalization factor $a$ discussed above.

Here, we detail the discrepancy between the spin wave mode and the low-lying excitation of the DSF for $K<0$, which is another clue to categorize the magnetic ordered phases into the categories I and II in addition to the temperature dependence of specific heat. When the system approaches the Kitaev's QSL phase around $\varphi=270^{\circ}$, the categorization of the categories I and II can be discussed from the discrepancy between the spin wave mode and the low-lying excitation of the DSF at the M point. At $\varphi=210^{\circ}$ in the category I, the low-lying excitation is well explained by the spin wave mode. However, the discrepancy between the low-lying excitation of the DSF and the spin wave mode develops clearly at the M point at $\varphi=240^{\circ}$ in the category II. The low-lying excitations of the DSF are located in the lower energy region than that of the spin wave mode. (The discrepancy between the both is also clear at the Y point. However, the excitation of the DSF at the $\mathrm{Y}$ point is identical to that at the $\mathrm{M}$ point. This is due to to the finite size effect; the symmetry breaking is prohibited in the finite size systems.) We regard $\varphi=210^{\circ}$ is located in I near in the crossover region of the categories I and II, where both characters are mixed. Based on these observations, here we categorize $\varphi=210^{\circ}$ and $\varphi=240^{\circ}$ as the category I and category II, respectively. In the stripy phase for $\varphi>270^{\circ}$, the wave vector, where the spin wave mode deviates from 
the low-lying excitations of the DSF, moves to the $\Gamma$ and $\Gamma^{*}$ points. At $\varphi=300^{\circ}$, the low-lying excitation of the DSF shows slight softening at the $\Gamma$ and $\Gamma^{*}$ points in comparison with the spin wave mode. In addition, the temperature dependence of $C$ shows a single peak with a prominent shoulder, which is in between the single peak structure of the category I and the two-peak structure of the category II. Consequently, the system at $\varphi=300^{\circ}$ is concluded to be located in the crossover region between the category I and the category II.

\section{B. Results for $a b$ initio Hamiltonian of $\mathrm{Na}_{2} \mathrm{IrO}_{3}$}

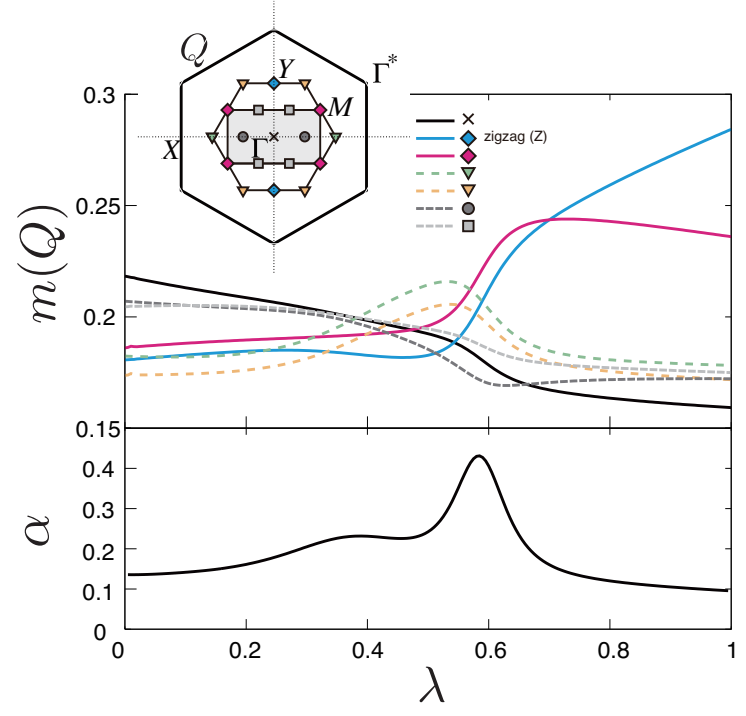

FIG. 6: (Color online) Square root of the equal-time spin structure factors, $m(\boldsymbol{Q})$ and the second derivative of ground state energy $\alpha$ as functions of $\lambda$ calculated with the $N=24$ site cluster in Fig[1(d).

In the light of the categorization examined in the generalized Kitaev-Heisenberg models, we examine the interpolated Hamiltonian between the Kitaev limit, $\hat{H}_{\lambda=0}$, and the $a b$ initio Hamiltonian of $\mathrm{Na}_{2} \mathrm{IrO}_{3}, \hat{H}_{\lambda=1}$, given in Eq.(5). First, we show that the peak value of the equaltime spin structure factor for the zigzag order starts growing at an onset value $\lambda_{c}$. Here $\lambda_{c}$ is around 0.6. For all $0 \leq \lambda \leq 1$, the dynamical spin structure factor of $\hat{H}_{\lambda}$ is not captured by the linear spin wave theory ${ }^{16}$. Next, we show that the temperature dependence of the specific heat for $\hat{H}_{\lambda}$ always has a two-peak structure irrespective of $\lambda$.

The quantum phases of the interpolated Hamiltonian are examined by equal-time spin structure factors and the second derivatives of the ground state energy defined below; here we introduce the square root of the normalized equal-time spin structure factor, which is extrapolated to the magnetic order parameter at momentum $\boldsymbol{Q}$ in the thermodynamic limit, defined as

$$
m(\boldsymbol{Q}) \equiv \lim _{T \rightarrow+0} \sqrt{S_{T}(\boldsymbol{Q})},
$$

where we take the $T=0$ limit.

The quantum phase transitions are expected to cause divergence or discontinuity (or a sharp peak in finite-size systems) in the second derivatives of the ground state energy $E$ with respect to $\lambda$,

$$
\alpha \equiv-\frac{d^{2} E / N}{d \lambda^{2}}
$$

In Fig 6 the $\lambda$-dependences of $m(\boldsymbol{Q})$ and $\alpha$ for $N=24$ site cluster are given.

From the growth of $m(\boldsymbol{Q})$ at the Y point corresponding to the zigzag order observed in the experiments and a peak in $\alpha$ at $\lambda \sim 0.59$, we conclude that the zigzag order appears for $\lambda \gtrsim 0.6$. For $\lambda \lesssim 0.4$, we expect the Kitaev's QSL ground states. Since the phase transition around $\lambda \sim 0.6$ appears to be continuous with the reduction of $m$ toward the transition point $\lambda \sim 0.6$, the distance from the Kitaev's QSL phase may be measured from the ordered moment.

The presence of the phase boundary to the zigzag ordered phase is also confirmed from the DSF results shown in Fig. 7 At $\lambda=0$, we observe a characteristic nondispersive mode at $\omega / A \sim 0.3$ reflecting the Kitaev's QSL ground state $e^{5}$. For $\lambda<0.6$, some poles with the weak intensity appear below $\omega / A \sim 0.3$ and the peak with the largest intensity is not located in the lowest excitation mode. These properties of the low-energy excitations are contrast to those in the magnetic ordered phase, where the lowest excitation usually shows the largest intensity. We also observe the lack of well developed peaks in the equal-time spin structure factors shown in Fig. 6. Thus, we conclude that the ground state at $\lambda<0.6$ is still the Kitaev's QSL state.

For $0.6<\lambda \leq 1.0$, the lowest excitation with the largest intensity appears at the $\mathrm{M}$ or $\mathrm{Y}$ point and the peak at the $\mathrm{Y}$ point develops as $\lambda$ increases. In this region, the equal-time spin structure factors at the $\mathrm{M}$ and Y point well develops as shown in Fig. [6] Therefore, the ground state becomes magnetically ordered for $\lambda>0.6$ and the presence of the phase boundary is expected for $\lambda \sim 0.6$.

To confirm that the interpolated Hamiltonian $\hat{H}_{\lambda}$ is categorized into the category II for $\lambda>0.6$, we also examine the temperature dependences of the specific heat $C$. The results are shown in Fig. 8. First of all, for the entire parameter range, $0 \leq \lambda \leq 1$, two peaks are seen in the temperature dependences of $C$. The low-temperature peak is at $T /|K| \lesssim 0.03$, and the higher (high- $T$ ) one is at $T /|K| \sim 0.3$, where $|K|=30.7 \mathrm{meV}(356 \mathrm{~K}$ ) (see Table III). As already discussed in Ref. 5 and Ref. 6, the origin of the high- $T$ peak is well explained by the growth of magnetic correlations for the nearest neighbor pairs, which is determined by the Kitaev couplings, $K \sim K^{\prime}$. The low- $T$ peak in the pure Kitaev model corresponds to 


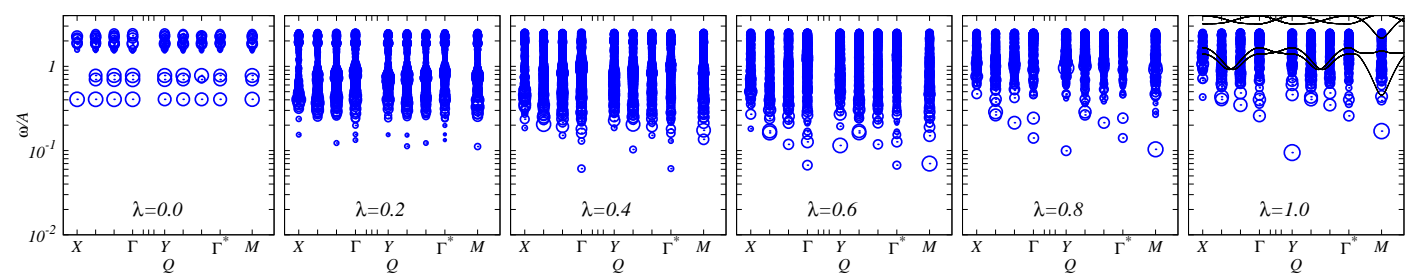

FIG. 7: (Color online) Dynamical spin structure factors of the interpolated Hamiltonian $\hat{H}_{\lambda}$ between the Kitaev limit and the $a b$ initio spin Hamiltonian proposed in Ref. 14. From the leftmost to rightmost panels, $S(\boldsymbol{Q}, \omega)$ is shown for $\lambda=0, \lambda=0.2$, $\lambda=0.4, \lambda=0.6, \lambda=0.8$, and $\lambda=1$. Abscissa represents the labeled points in Fig 5 (i).

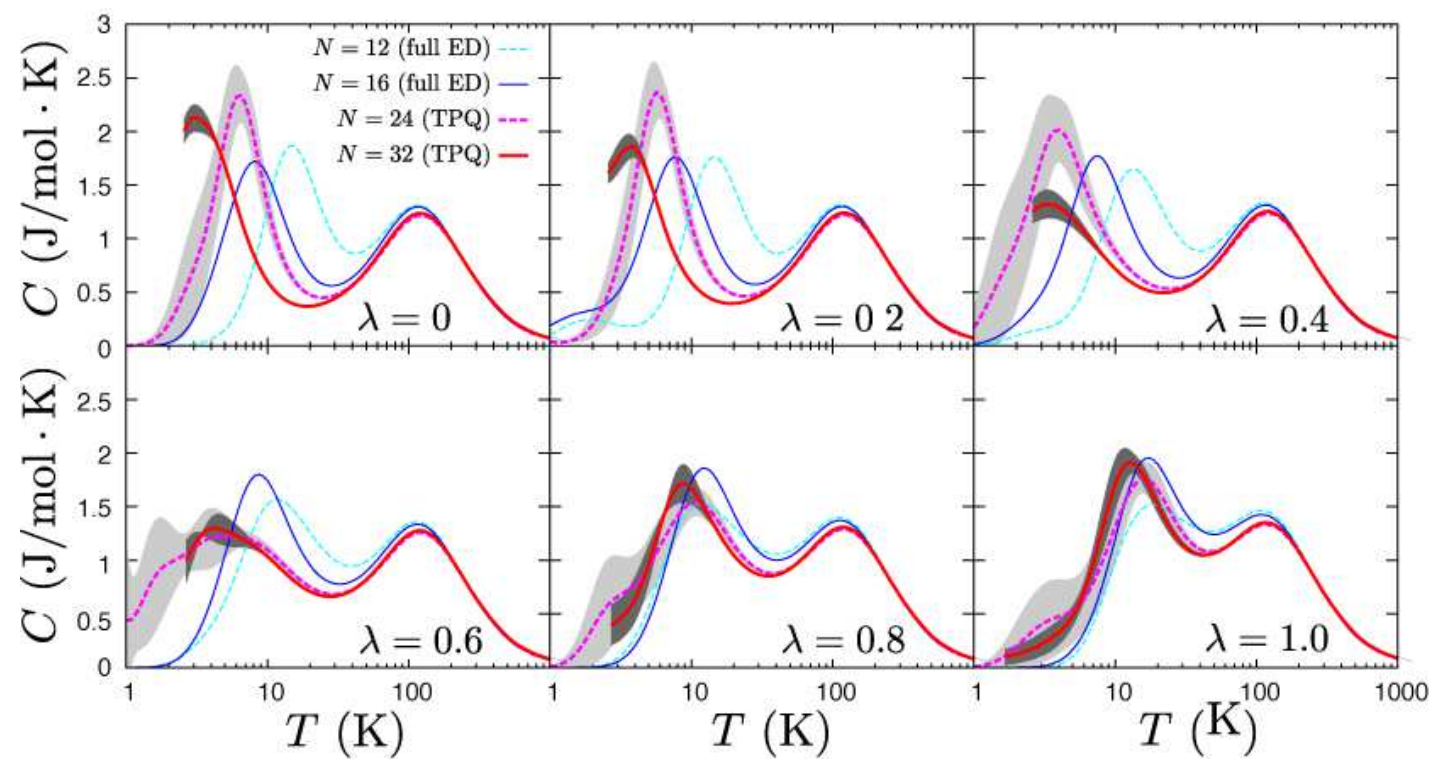

FIG. 8: (Color online) Temperature dependences of the specific heat $C$ of the interpolated Hamiltonian $\hat{H}_{\lambda}$ between the Kitaev limit and the $a b$ initio spin Hamiltonian proposed in Ref. 14. The results for $N=12$ and $N=16$ obtained by fully diagonalizing $\hat{H}_{\lambda}$ (denoted as "full ED") are illustrated with thin broken and thin solid (blue) curves, respectively. For $N=24$ and $N=32$ (thick broken and thick solid (red) curves), the thermal pure quantum (TPQ) states ${ }^{20}$ are employed. The possible errors of TPQ due to the truncation of the Hilbert space are shown in $C$ by shaded (gray) belts, which is estimated by using the standard deviation of the results obtained from 4 to 36 initial random wave functions.

the thermal fluctuation of the local $Z_{2}$ gauge field that is one of two Majorana fermions yielded via the fractionalization of an original quantum spin. The non-monotonic $\lambda$-dependences in the low- $T$ peak correspond to the quantum phase transition around $\lambda \sim 0.6$ and possible emergence of the intermediate phase for $0.4 \lesssim \lambda \lesssim 0.6$.

\section{DISTANCE FROM THE KITAEV SPIN LIQUID PHASE}

In the previous section, Sec. III, magnetically ordered materials categorized as the category II are expected to be close to the Kitaev's spin liquid in the parameter space of the effective Hamiltonians. We further propose more quantitative measure to estimate the distance between the real material and the Kitaev's QSL.

As is shown in Figs. 2 and 8 the specific heat $C$ in the category II shows two peaks similar to the category III. However, the two-peak structure in the temperature dependence of $C$ itself is not a unique feature in the vicinity of the Kitaev's QSL. For example, geometrically frustrated and quasi-one-dimensional quantum spin systems also show the two-peak structure in the temperature dependence of $C \underline{\underline{26}}$. In that case, the high- and low-temperature peaks depend on the dimensional anisotropy, where the low-temperature peak in $C$ represents the entropy release arising from the real longrange order. If the anisotropy increases, the release of the entropy at the low temperature peak may decrease. 


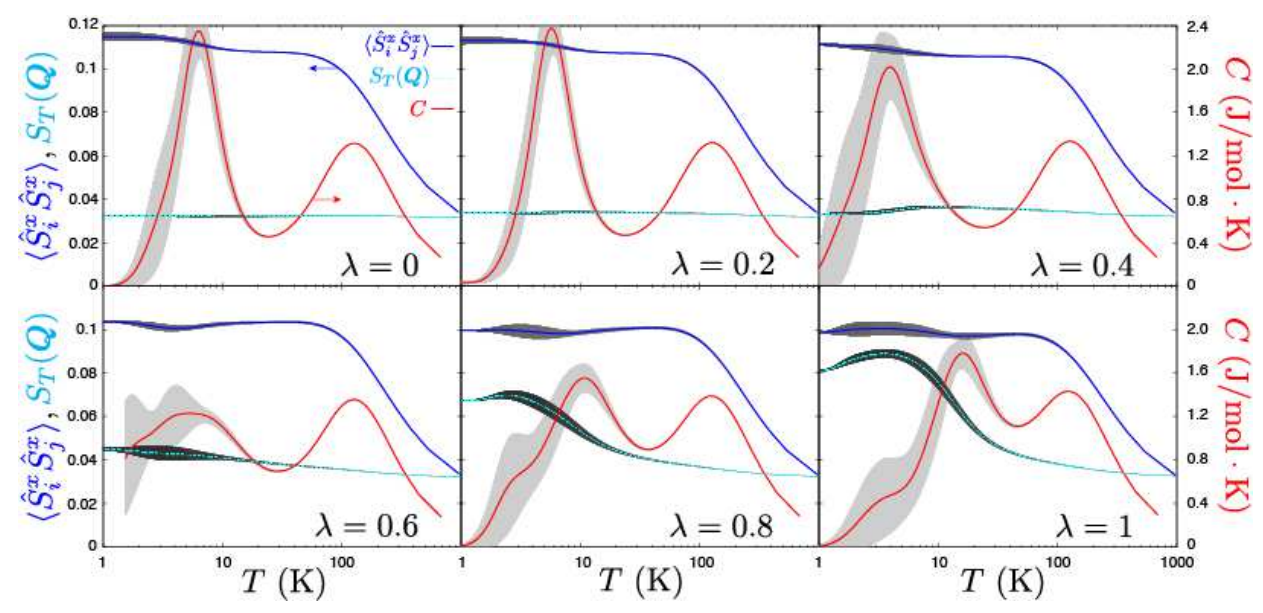

FIG. 9: (Color online) Temperature dependence of the short-range spin correlation $\left\langle\hat{S}_{i}^{x} \hat{S}_{j}^{x}\right\rangle_{T}$ for $X$-bond and the long-range spin correlation $S_{T}(\boldsymbol{Q})$ in comparison with the specific heat $C$. The results for $N=24$ by employing the thermal pure quantum states $^{20}$ are shown. The possible errors of TPQ due to the truncation of the Hilbert space are shown in $\left\langle\hat{S}_{i}^{x} \hat{S}_{j}^{x}\right\rangle_{T}, S_{T}(\boldsymbol{Q})$, and $C$ by shaded (gray) belts, which is estimated by using the standard deviation of the results obtained from 4 to 36 initial random wave functions. For $S_{T}(\boldsymbol{Q})$, the Y point is selected as the wave vector $\boldsymbol{Q}$, which corresponds to the zigzag (Z) order ${ }^{14}$.

On the other hand, the two-peak structure of $C$ in the Kitaev's QSL originates from the fractionalization of the spin degrees of freedom. The fractionalization is expected to be more evident in temperature dependence of the entropy $S$ that directly shows the fractionalized spin degrees of freedom. A quantitative way of measuring the distance to the Kitaev's QSL is to observe the temperature dependences of $S$. As clarified in Ref. 6, at temperatures between the two peaks of $C$, the systems with the Kitaev's QSL ground states show a half plateau in $S$ with the value $\left(N k_{B} / 2\right) \ln 2$. The value $\left(N k_{B} / 2\right) \ln 2$ is not generically expected in other cases unless some accidental coincidence happens. Thus, this value is a hallmark of the Kitaev's QSL and persists in the systems in close vicinity of the Kitaev's QSL phase, even when the systems show magnetically ordered states, as shown in the remaining part.

Before discussing the temperature dependence of the entropy $S$, we explain the physical mechanism of the twopeak formation in $C$ from the viewpoint of the spin correlation. The growth of the long-range spin correlations below the low-temperature peak of $C$ clearly distinguishes the magnetic ordered ground states in the category II from the Kitaev's QSL in the category III, as seen in Figs. 4 and 9 For example, in the generalized KitaevHeisenberg Hamiltonian with $\varphi=100^{\circ}$ and $\varphi=240^{\circ}$ and in the ab initio Hamiltonian for $\lambda \gtrsim 0.6, S_{T}(\boldsymbol{Q})$ grows with decreasing temperature and is saturated only below the lower temperature peak, while that remains small in the Kitaev's QSL phase. On the other hand, the shortrange spin correlations grows with decreasing temperatures until the saturation below the high-temperature peak of $C$ for the entire parameter ranges.
We show the temperature dependences of $S$ in Fig 10 for the generalized Kitaev-Heisenberg Hamiltonian. When the system is categorized as the categories II or III, a shoulder around $S=\left(N k_{B} / 2\right) \ln 2$ is observed in the temperature dependence of $S$. At least, the value $\left(N k_{B} / 2\right) \ln 2$ is due to the contribution from the free Majorana fermions in the category III. The plateau in $S$ is evident at an anisotropic limit $\left|K_{z}\right| \gg\left|K_{x}\right|=\left|K_{y}\right|$ $\left(K \gg K^{\prime}\right)^{6}$, and is smeared out as the system approaches the symmetric Kitaev couplings, $\left|K_{z}\right|=\left|K_{x}\right|=\left|K_{y}\right|$ $\left(K \sim K^{\prime}\right)$, which is identical to $\varphi=90^{\circ}\left(\varphi=270^{\circ}\right)$. However, the remaining feature, namely the shoulder structure, is still an evidence for the fractionalization of the quantum spins. In the present case, we still observe such shoulder structure of $S$ even in the category II, if the system is located in the vicinity of the Kitaev's QSL phase.

To confirm the existence of the shoulder around $S=$ $\left(N k_{B} / 2\right) \ln 2$ more quantitatively, we decompose the temperature dependence of $S / N k_{B} \ln 2$ by employing a phenomenological fitting function,

$$
\sigma(T)=\sum_{\ell=1,2} \tau_{\ell}(T)
$$

where $\tau_{\ell}(T)$ is defined as

$$
\tau_{\ell}(T)=\frac{\rho_{\ell} / 2}{1+\exp \left[\left(\frac{\beta_{\ell}+\gamma_{\ell} T_{0 \ell} / T}{1+T_{0 \ell} / T}\right) \ln \left(\frac{T_{0 \ell}}{T}\right)\right]},
$$

with constraints $T_{01} \geq T_{02}$ and $\rho_{1}+\rho_{2}=2$. The specific functions $\tau_{\ell}(T)$ are chosen to describe power-low asymptotic behaviors at both of low and high temperature limits, as detailed in Appendix A. As shown in Fig. 11. 


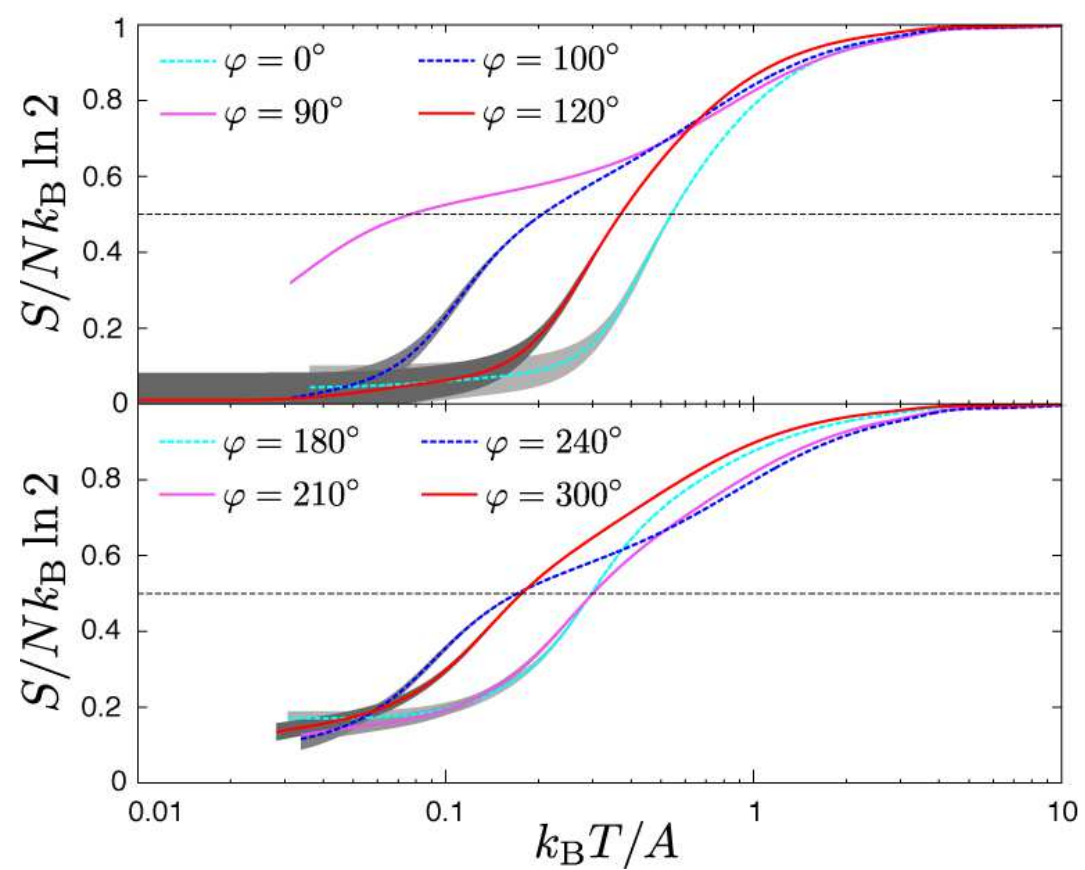

FIG. 10: (Color online) Temperature dependence of the entropy for the generalized Kitaev-Heisenberg Hamiltonian. The horizontal broken line indicates $S=\left(N k_{B} / 2\right) \ln 2$. The possible errors of TPQ due to the truncation of the Hilbert space are shown in $S$ by shaded (gray) belts, which is estimated by using the standard deviation of the results obtained from 4 initial random wave functions.

we successfully decompose $S$ of $\hat{H}_{\mathrm{CJK}}$ at $\varphi=90^{\circ}$ into two components. Here, we employ the standard least square fitting of the expectation values of $S / N k_{B} \ln 2$ for $N=32$, with the fitting function $\sigma(T)$. The ratio of the weight for two parts, $\tau_{\ell}(T)$, satisfies $\rho_{1} / \rho_{2} \sim 1$ in the Kitaev's QSL phase at $\varphi=90^{\circ}$, as expected, and even in the category II. When the system goes toward deep inside of the magnetic ordered phase of the category I, the shoulder in $S$ continuously varies and finally disappears. From Fig. 12, we confirm that the ratio $\rho_{1} / \rho_{2}$ also largely deviates from 1 in the category I. Therefore, the successful fitting with $\rho_{1} / \rho_{2} \sim 1$ in the category II supports that the system is located in the vicinity of the Kitaev's QSL phase. Here, we note that, in Fig. 12, due to $2 S+1$-fold ground-state degeneracy in the ferromagnetic phase at $\varphi=180^{\circ}$ with the total spin $S_{\text {tot }}=N / 2, \rho_{1}$ and $\rho_{2}$ are rescaled by a factor $\left[1-(N \ln 2)^{-1} \ln \left(2 S_{\text {tot }}+1\right)\right]^{-1}$ with $N=32$ and the total spin $S_{\text {tot }}=N / 2=16$.

We apply the same analysis to the $a b$ initio Hamiltonian case. In Fig 13, we show temperature dependences of $S$. The two-peak structure of $C$ is common for the entire parameter range, $0 \leq \lambda \leq 1$, and the plateau or shoulder around $S=\left(N k_{B} / 2\right) \ln 2$ is always observed. At least, for $\lambda=0$, the value $\left(N k_{B} / 2\right) \ln 2$ corresponds to the contribution of the free Majorana fermions obviously, because the system is in the Kitaev's QSL phase.

As shown in Fig 14, we also successfully decompose $S$ into two components for the ab initio model, $\lambda=1$.
The obtained fitting parameters are $\beta_{1}=1.74 \pm 0.04$, $\gamma_{1}=1.88 \pm 0.09, T_{01}=120 \pm 2 \mathrm{~K}, \beta_{2}=2.26 \pm 0.06, \gamma_{2}=$ $2.64 \pm 0.01, T_{02}=13.6 \pm 0.1 \mathrm{~K}$, and $\rho_{1}=0.99 \pm 0.02$ with the constraint $\rho_{2}=2-\rho_{1}$. This successful fitting also supports that the shoulder structure in the temperature dependences of $S$ is a hallmark that $\mathrm{Na}_{2} \mathrm{IrO}_{3}$ is located in the vicinity of the Kitaev's QSL phase. As expected from the successful fitting for $\lambda=1$, the decomposition is also successful for $0 \leq \lambda<1$ with $\rho_{1} / \rho_{2} \sim 1$, as shown in Fig [15]

After confirming the shoulder structure around $S=$ $\left(N k_{B} / 2\right) \ln 2$, a quantitative measure for distance between the target system in the category II and the Kitaev's QSL can be introduced: The ratio of $T_{\ell}$ to $T_{h}$ gives a quantitative measure for the distance, where $T_{\ell}\left(T_{h}\right)$ is the location of the low-temperature (high-temperature) peak of the specific heat $C$. Details in the estimation of $T_{\ell}$ and $T_{h}$ are given in Appendix B The two temperature scales $T_{01}$ and $T_{02}$ used in Eq.(23) roughly agree with $T_{h}$ and $T_{\ell}$, respectively. From the results of $C$ for $N=32$ shown in Fig 8 the ratio $T_{\ell} / T_{h}$ is smaller than 0.03 for the Kitaev's QSLs. By taking into account the $N$-dependence of $T_{\ell}$, namely, the monotonic decreases in $T_{\ell}$ as $N$ increases, in the category III, the condition $T_{\ell} / T_{h}=0.03$ gives the upper limit on the ratio $T_{\ell} / T_{h}$ of the Kitaev's QSLs.

In Fig 16, the ratio $T_{\ell} / T_{h}$ obtained through the fitting is summarized. As shown in Fig. 8 , for $\lambda=1$, 


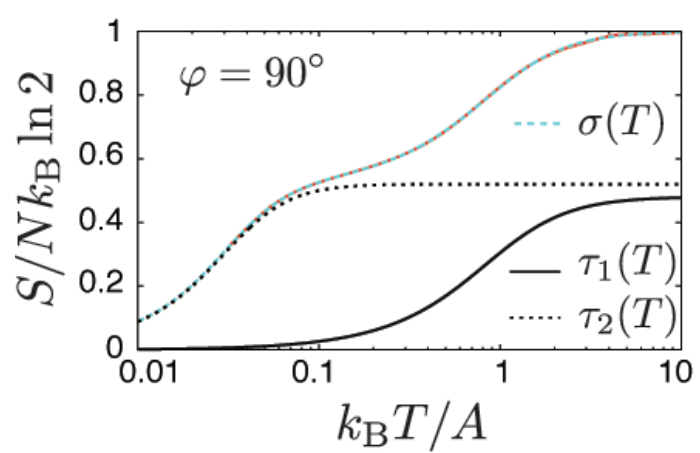

FIG. 11: (Color online) Decomposition of $S$ for the generalized Kitaev-Heisenberg Hamiltonian with $\varphi=90^{\circ}$. The possible errors of TPQ due to the truncation of the Hilbert space are shown in $S$ by shaded (gray) belts, which is estimated by using the standard deviation of the results obtained from 4 initial random wave functions. The function $\sigma(T)\left(=\tau_{1}(T)+\tau_{2}(T)\right)$ defined in Eqs. (22) and (23) is employed to fit the numerical result denoted by the (red) solid curve. The fitting function $\sigma(T)$ denoted by the broken (light blue) curve is almost on top of the numerical result of $S$ for $\varphi=90^{\circ}$. The decomposed components $\tau_{1}(T)$ and $\tau_{2}(T)$ are shown in (black) solid and dotted curves, respectively.

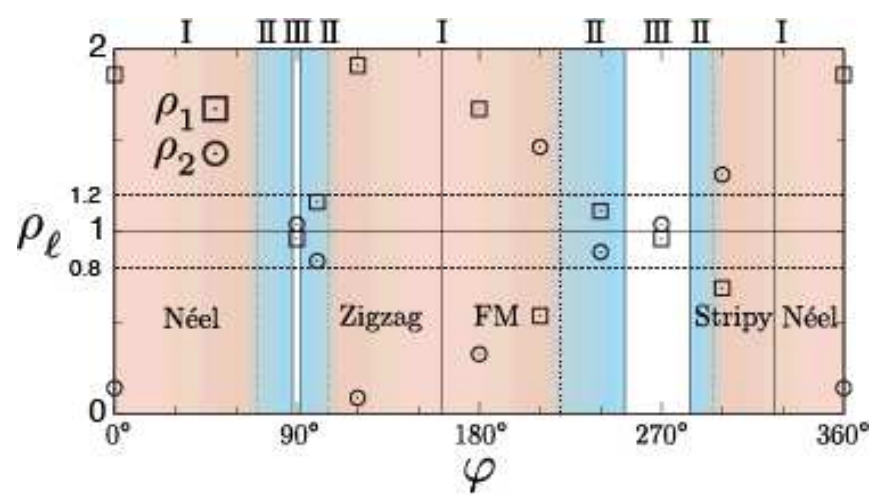

FIG. 12: (Color online) Weights $\rho_{1}$ and $\rho_{2}$ in least-square fitting of $S(T) / N k_{B} \ln 2$ with $\sigma(T)$ as functions of $\varphi$.

the $N$-dependence is almost converged up to $N=32$. Therefore, the estimate on the ratio $T_{\ell} / T_{h} \sim 0.11$ offers a prediction for experimental observations, which simultaneously offers experimental test on the $a b$ initio Hamiltonian for $\mathrm{Na}_{2} \mathrm{IrO}_{3}$. In the inset of Fig 16 , the schematic phase diagram expected at the thermodynamic limit is shown. From development of spin correlations shown in Fig 9 the transition temperatures $T_{N}$ below which the zigzag orders set in are expected just below the low temperature scale $T_{\ell} \sim T_{02}$. Note that the present two-dimensional $a b$ initio Hamiltonian has a magnetic anisotropy. Thus, it is expected to show a finite-temperature spontaneous symmetry breaking in the thermodynamic limit.

\section{DISCUSSION}

\section{A. Isoelectric doping and new materials}

There exist experimental attempts to realize the Kitaev's spin liquid by isoelectronic doping starting with $\mathrm{Na}_{2} \mathrm{IrO}_{3}$. However, these attempts are not so successful so far, as explained below.

Besides a search for new materials such as $\mathrm{Li}_{2} \mathrm{RhO}_{3} \underline{27,28}$ and $\alpha-\mathrm{RuCl}_{3} \stackrel{29}{\underline{31}}, \quad\left(\mathrm{Na}_{1-x} \mathrm{Li}_{x}\right)_{2} \mathrm{IrO}_{3}$ interpolating $\mathrm{Na}_{2} \mathrm{IrO}_{3}$ and $\mathrm{Li}_{2} \mathrm{IrO}_{3}$ has been studied $^{32,33}$. In Ref 32 , the Néel temperature $T_{N}$ is reported to be minimized around $x \sim 0.7$ and, simultaneously, the frustration parameter defined as the ratio of the Curie-Weiss constant $\Theta$ and $T_{N}, \Theta / T_{N}$, becomes maximum. The low temperature scale $T_{\ell}$ may also show minimum around $x \sim 0.7$, which seemingly suggests that $\left(\mathrm{Na}_{0.3} \mathrm{Li}_{0.7}\right)_{2} \mathrm{IrO}_{3}$ is a good candidate of the Kitaev's spin liquid.

However, we should note that there are several remaining issues in $\left(\mathrm{Na}_{1-x} \mathrm{Li}_{x}\right)_{2} \mathrm{IrO}_{3}$ as a hunting field of the Kitaev's spin liquid. First of all, phase separation for, at least, $0.25 \lesssim x \lesssim 0.6$ is reported and stability around $x \sim 0.7$ has not been confirmed yet. Second, the reported specific heat coefficient $C / T$ around the lowtemperature peaks seems too small around $x \sim 0.7$ to exhaust $\left(N k_{B} / 2\right) \ln 2$. An excess entropy release that reduces $C / T$ at low temperatures may be attributed to distortion due to the isoelectronic doping. Our ab initio studies suggest that $\left(\mathrm{Na}_{1-x} \mathrm{Li}_{x}\right)_{2} \mathrm{IrO}_{3}$ has a smaller lattice constant for larger $x$ because of smaller ionic radius of Li. This may enhance the further neighbor transfers that are harmful for realizing the Kitaev's spin liquid.

\section{B. Thin films}

Making thin films of $\mathrm{Na}_{2} \mathrm{IrO}_{3}$ and related materials on various substrates is another unexplored but promising approach to realize the Kitaev's spin liquid. For example, making thin films of a perovskite iridate $\mathrm{CaIrO}_{3}$ is efficient to stabilize the perovskite crystal structure unstable as a bulk crystal ${ }^{34}$, and is demonstrated to change the lattice constant depending on the substrates. Thin films of $\mathrm{Na}_{2} \mathrm{IrO}_{3}$ on an appropriate substrate may expand the lattice constant in comparison to the bulk $\mathrm{Na}_{2} \mathrm{IrO}_{3}$, which may decrease the other exchange couplings relative to the Kitaev exchange. This effectively reduces $\lambda$ and would stabilize the Kitaev's spin liquid. A combinatorial specific-heat measurement over the thin films of $\mathrm{Na}_{2} \mathrm{IrO}_{3}$ and related materials on various substrates is highly desirable although specific-heat measurements of thin films that need sophisticated micocalorimeters ${ }^{35}$ are not easy to carry out. Our criteria for the closeness to the Kitaev's 


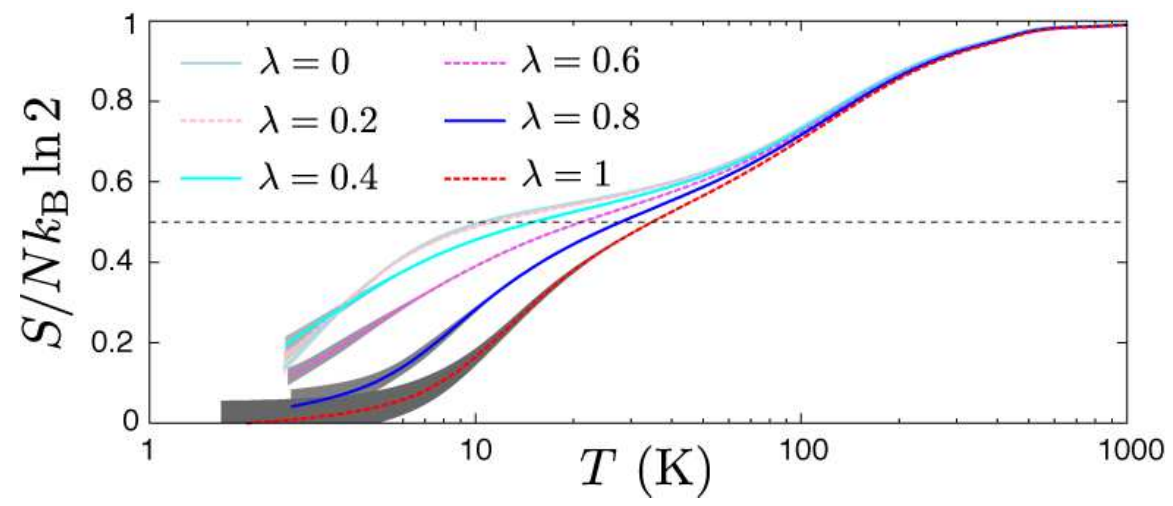

FIG. 13: (Color online) Temperature dependence of the entropy for the interpolated Hamiltonian. $\lambda=0(\lambda=1)$ corresponds to the Kitaev limit (the ab initio Hamiltonian of $\mathrm{Na}_{2} \mathrm{IrO}_{3}$ ). The results for $N=32$ are shown. The horizontal broken line indicates $S=\left(N k_{B} / 2\right) \ln 2$. The possible errors of TPQ due to the truncation of the Hilbert space are shown in $S$ by shaded (gray) belts, which is estimated by using the standard deviation of the results obtained from 4 initial random wave functions.

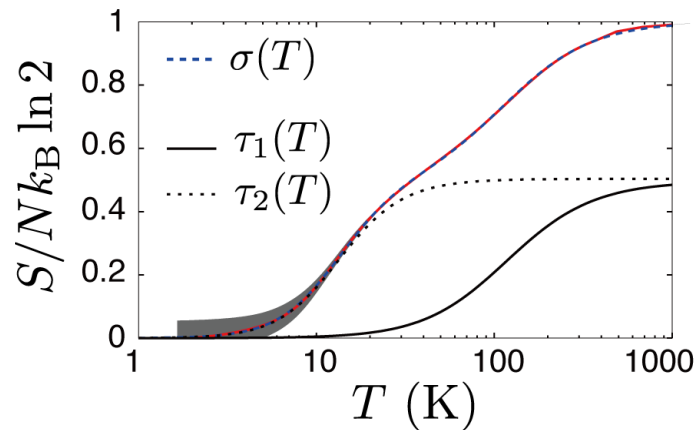

FIG. 14: (Color online) Decomposition of $S$ for the interpolated Hamiltonian with $\lambda=1$. The possible errors of TPQ due to the truncation of the Hilbert space are shown in $S$ by shaded (gray) belts, which is estimated by using the standard deviation of the results obtained from 4 initial random wave functions. The function $\sigma(T)\left(=\tau_{1}(T)+\tau_{2}(T)\right)$ defined in Eqs. (22) and (23) is employed to fit the numerical result denoted by the (red) solid curve. The fitting function $\sigma(T)$ denoted by the broken (light blue) curve is almost on top of the numerical result of $S$ for $\varphi=90^{\circ}$. The decomposed components $\tau_{1}(T)$ and $\tau_{2}(T)$ are shown in (black) solid and dotted curves, respectively.

spin liquid may help and point to the favorable direction of efforts.

\section{SUMMARY}

We have studied the magnetic excitations and specific heat of the generalized Kitaev-Heisenberg model and the ab initio effective Hamiltonian of $\mathrm{Na}_{2} \mathrm{IrO}_{3}$. By comparing the linear spin wave dispersion, the dynamical spin structure factors, temperature dependences of the spe-

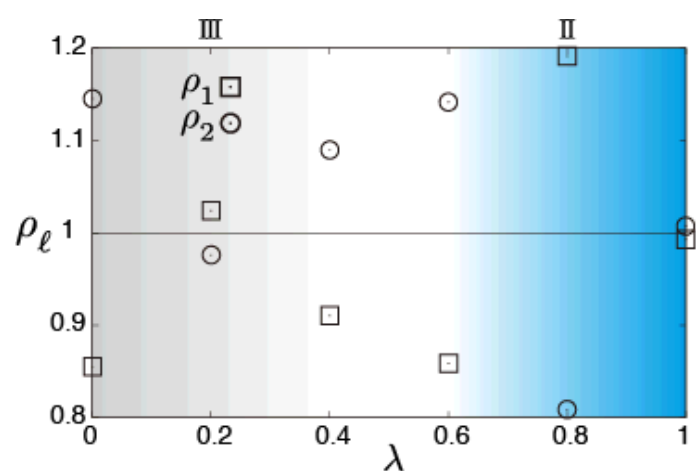

FIG. 15: (Color online) Weights $\rho_{1}$ and $\rho_{2}$ in least-square fitting of $S(T) / N k_{B} \ln 2$ with $\sigma(T)$ as functions of $\lambda$.

cific heat, we found that the parameter space of the effective Hamiltonians for $\mathrm{Na}_{2} \mathrm{IrO}_{3}$ can be classified to three categories; the phase diagram of the generalized KitaevHeisenberg model exhibits two qualitatively distinct regions in the magnetically ordered phase in addition to the Kitaev's spin liquid phase.

In one region of the magnetically ordered phase, the specific heat has two-peak structure. In addition, the conventional linear spin wave theory fails in explaining the low-lying excitation the dynamical spin structure factors and the half-plateau-like temperature dependences of the entropy is observed owing to the thermal fractionalization of the spin degrees of freedom. The other is the trivial region located far from the Kitaev's QSL. In the trivial region, we observe the low-lying excitation well explained by linear spin wave theory and the single peak structure in the temperature dependence of the specific heat.

The $a b$ initio Hamiltonian of $\mathrm{Na}_{2} \mathrm{IrO}_{3}$, whose ground 


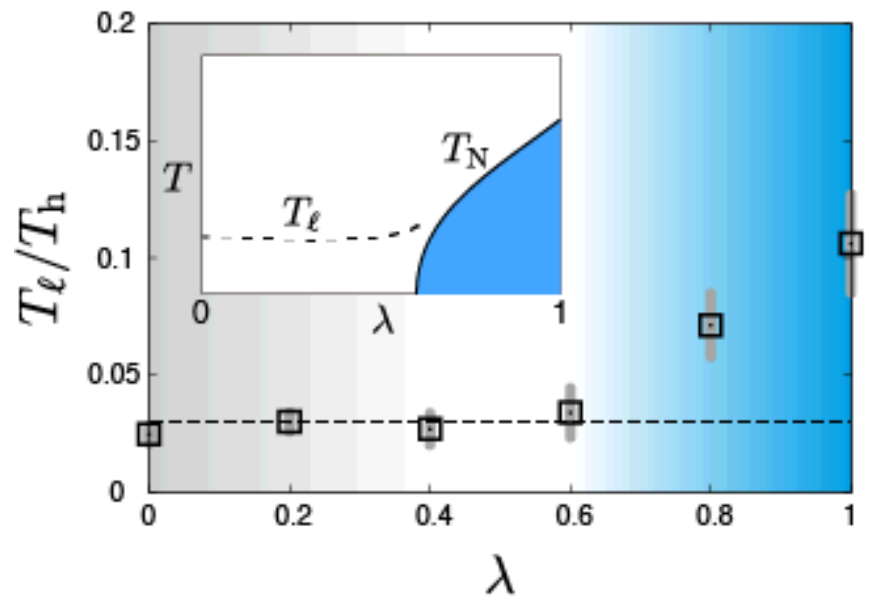

FIG. 16: (Color online) Ratio of two temperature scales, $T_{\ell}$ and $T_{h}$. The ratio $T_{\ell} / T_{h}(<1)$ is obtained through the fitting function $\sigma(T)$ defined in Eqs.(22) and (23). The horizontal broken line represents a proposed upper bound for $T_{\ell} / T_{h}$ in the Kitaev's spin liquids. The vertical (grey) bars illustrate uncertainty in $T_{\ell} / T_{h}$ due to uncertainty in $C(T)$. The shaded areas for $\lambda \gtrsim 0.6$ and $\lambda \lesssim 0.4$ indicate the zigzag ordered and the Kitaev's QSL ground states, respectively. The inset shows schematic phase diagram expected from the present results. Here, the temperature $T_{\ell}$ corresponds to the lower temperature peak of $C$ in Fig 8 which is denoted as the broken curve. The $\lambda$-dependence of the expected Néel temperature $T_{N}$ for the zigzag ordered phase is shown in the solid curve.

state is the zigzag magnetic order, indeed shows the two peaks in the specific heat and indicates the breakdown of the linear spin wave theory and the half-plateau-like temperature dependences of the entropy pinned around $\left(N k_{B} / 2\right) \ln 2$, which signals that the ab initio Hamiltonian of $\mathrm{Na}_{2} \mathrm{IrO}_{3}$ is in the vicinity of the Kitaev's QSL phase. These theoretical distinctions between the trivially ordered ground state and the system close to the Kitaev's QSL, offer experimental clues and criteria to understand a given material in terms of the distance from the Kitaev's QSL phase. It also offers a guideline for experiments to search the Kitaev materials.

\section{Acknowledgments}

We thank T. Okubo, and T. Tohyama for fruitful discussions. This work was supported by the Computational Materials Science Initiative (CMSI), and KAKENHI(Grants No. 25287104, 25287097, 15K05232, 15K17702, and No. 25287088) from MEXT Japan. We thank the computational resources of the $\mathrm{K}$ computer provided by the RIKEN Advanced Institute for Computational Science through the HPCI System Research project (hp120283, hp130081, hp140215 and hp150211). We also thank numerical resources in the ISSP Supercomputer Center at University of Tokyo and the Research
Center for Nano-micro Structure Science and Engineering at University of Hyogo.

\section{Appendix A: Decomposition of entropy $S(T)$}

Decomposition of $S(T)$ is examined to capture a sign of the thermal fractionalization in the vicinity of the Kitaev spin liquids. If the system is categorized into the category II or III, $S(T)$ is decomposed into two parts with nearly equal weights. Although the temperature dependence of $S(T)$ is complicated in general, a simple ansatz defined below works as shown in Fig 11 and Fig 14 .

To formulate our ansatz on the decomposition of $S(T)$, we recall high-temperature and low-temperature behaviors of the entropy in simple systems. As the simplest example of temperature dependence of entropy, the Schottky entropy,

$$
\begin{aligned}
S_{\mathrm{S}}(T) & =\int_{0}^{T} d T^{\prime} \frac{C_{\mathrm{S}}\left(T^{\prime}\right)}{T^{\prime}} \\
& =\ln \left(1+e^{T_{0} / T}\right)-\frac{T_{0}}{T} \frac{e^{T_{0} / T}}{1+e^{T_{0} / T}},
\end{aligned}
$$

with a characteristic temperature $T_{0}$ gives a typical hightemperature behavior as $S_{\mathrm{S}}(T) \simeq \ln 2-\left(T_{0} / T\right)^{2} / 8$ for $T_{0} / T \ll 1$. In addition to essentially singular behaviors in $S_{\mathrm{S}}(T)$ due to excitation gaps, spin-wave-like power-law behaviors such as, $S_{\mathrm{S}}(T) \propto T^{2}+\mathcal{O}\left(T^{3}\right)$, are important at the low-temperature limit. To capture the power-law behaviors, instead of Eq. A1 , we assume the following function to fit our numerical results of $S(T)$ :

$$
\sigma(T)=\sum_{\ell=1,2} \tau_{\ell}(T),
$$

where

$$
\tau_{\ell}(T)=\left(\rho_{\ell} / 2\right) /\left[1+\left(T_{0 \ell} / T\right)^{p_{\ell}\left(T / T_{0 \ell}\right)}\right],
$$

with smooth functions $p_{\ell}(x)$. To interpolate two powerlaw behaviors at high- and low-temperature limits and to mimic gap-like behaviors, we employ one of the simplest rational form as

$$
p_{\ell}\left(T / T_{0 \ell}\right)=\frac{\beta_{\ell}+\gamma_{\ell} T_{0 \ell} / T}{1+T_{0 \ell} / T},
$$

where $\beta_{\ell}$ and $\gamma_{\ell}$ correspond to exponents in the high- and low-temperature power-law behaviors, respectively.

\section{Appendix B: Ratio $T_{\ell} / T_{h}$}

In this Appendix, details are given for the prescription how to determine the temperature scales, $T_{\ell}$ and $T_{h}$, and their error bars. The two temperature scales $T_{\ell}$ and $T_{h}$ of $\hat{H}_{\lambda}$ are simply determined as those of the peaks in the specific heat $C(T)$ for the largest system 
size, $N=32$, in the present paper. The higher temperature scale $T_{h}$ is determined with negligibly small uncertainty. The lower temperature scale $T_{\ell}$ is, however, inevitably under a numerical uncertainty in $C(T), \delta C(T)$, at low temperatures. Thus, here, we estimate errors by expanding $C(T)$ with respect to $T-T_{\ell}$ around $T=T_{\ell}$ as $C(T) \simeq C\left(T_{\ell}\right)-R\left(T-T_{\ell}\right)^{2}$. Then, the uncertainty in $T_{\ell}$ is naturally estimated as $\delta T_{\ell}=(\overline{\delta C} / R)^{1 / 2}$ with the upper bound of $\delta C(T), \overline{\delta C}=\max _{T}\{\delta C(T)\}$. The ratio of the temperature scales $T_{\ell} / T_{h}$ is shown in Fig 16 with the error bars.
1 P. W. Anderson, Mater. Res. Bull. 8, 153 (1973).

2 P. Fazes and P. W. Anderson, Philos. Mag. 30, 423 (1973).

3 A. Kitaev, Annals Phys. 321, 2 (2006).

${ }^{4}$ G. Baskaran, S. Mandal, and R. Shankar, Phys. Rev. Lett. 98, 247201 (2007).

5 J. Knolle, D. L. Kovrizhin, J. T. Chalker, and R. Moessner, Phys. Rev. Lett. 112, 207203 (2014).

6 J. Nasu, M. Udagawa, and Y. Motome, Phys. Rev. B 92, $115122(2015)$.

7 G. Jackeli and G. Khaliullin, Phys. Rev. Lett. 102, 017205 (2009).

8 J. c. v. Chaloupka, G. Jackeli, and G. Khaliullin, Phys. Rev. Lett. 105, 027204 (2010).

9 J. c. v. Chaloupka, G. Jackeli, and G. Khaliullin, Phys. Rev. Lett. 110, 097204 (2013).

10 S. K. Choi, R. Coldea, A. N. Kolmogorov, T. Lancaster, I. I. Mazin, S. J. Blundell, P. G. Radaelli, Y. Singh, P. Gegenwart, K. R. Choi, et al., Phys. Rev. Lett. 108, 127204 (2012).

11 F. Ye, S. Chi, H. Cao, B. C. Chakoumakos, J. A. Fernandez-Baca, R. Custelcean, T. F. Qi, O. B. Korneta, and G. Cao, Phys. Rev. B 85, 180403 (2012).

12 I. Kimchi and Y.-Z. You, Phys. Rev. B 84, 180407 (2011).

13 V. M. Katukuri, S. Nishimoto, V. Yushankhai, A. Stoyanova, H. Kandpal, S. Choi, R. Coldea, I. Rousochatzakis, L. Hozoi, and J. van den Brink, New Journal of Physics 16, 013056 (2014).

14 Y. Yamaji, Y. Nomura, M. Kurita, R. Arita, and M. Imada, Phys. Rev. Lett. 113, 107201 (2014).

15 Y. Sizyuk, C. Price, P. Wölfle, and N. B. Perkins, Phys. Rev. B 90, 155126 (2014).

16 T. Suzuki, T. Yamada, Y. Yamaji, and S.-i. Suga, Phys. Rev. B 92, 184411 (2015).

17 J. G. Rau and H.-Y. Kee, arXiv:1408.4811.

18 J. c. v. Chaloupka and G. Khaliullin, Phys. Rev. B 92, 024413 (2015).

19 A. Banerjee, C. Bridges, J. Yan, A. Aczel, L. Li, M. Stone, G. Granroth, M. Lumsden, Y. Yiu, J. Knolle, et al.,
arXiv:1504.08037.

20 S. Sugiura and A. Shimizu, Phys. Rev. Lett. 108, 240401 (2012).

21 S. Sugiura and A. Shimizu, Phys. Rev. Lett. 111, 010401 (2013).

22 E. R. Gagliano and C. A. Balseiro, Phys. Rev. Lett. 59, 2999 (1987).

23 E. Dagotto, Rev. Mod. Phys. 66, 763 (1994).

24 J. des Cloizeaux and J. J. Pearson, Phys. Rev. 128, 2131 (1962).

25 R. R. P. Singh, Phys. Rev. B 39, 9760 (1989).

26 V. Hardy, S. Lambert, M. R. Lees, and D. McK. Paul, Phys. Rev. B 68, 014424 (2003).

27 Y. Luo, C. Cao, B. Si, Y. Li, J. Bao, H. Guo, X. Yang, C. Shen, C. Feng, J. Dai, et al., Phys. Rev. B 87, 161121 (2013).

28 I. I. Mazin, S. Manni, K. Foyevtsova, H. O. Jeschke, P. Gegenwart, and R. Valentí, Phys. Rev. B 88, 035115 (2013).

29 K. W. Plumb, J. P. Clancy, L. J. Sandilands, V. V. Shankar, Y. F. Hu, K. S. Burch, H.-Y. Kee, and Y.-J. Kim, Phys. Rev. B 90, 041112 (2014).

30 J. A. Sears, M. Songvilay, K. W. Plumb, J. P. Clancy, Y. Qiu, Y. Zhao, D. Parshall, and Y.-J. Kim, Phys. Rev. B 91, 144420 (2015).

31 Y. Kubota, H. Tanaka, T. Ono, Y. Narumi, and K. Kindo, Phys. Rev. B 91, 094422 (2015).

32 G. Cao, T. F. Qi, L. Li, J. Terzic, V. S. Cao, S. J. Yuan, M. Tovar, G. Murthy, and R. K. Kaul, Phys. Rev. B 88, 220414 (2013).

33 S. Manni, S. Choi, I. I. Mazin, R. Coldea, M. Altmeyer, H. O. Jeschke, R. Valentí, and P. Gegenwart, Phys. Rev. B 89, 245113 (2014).

34 D. Hirai, J. Matsuno, D. Nishio-Hamane, and H. Takagi, Applied Physics Letters 107, 012104 (2015).

35 F. Giazotto, T. T. Heikkilä, A. Luukanen, A. M. Savin, and J. P. Pekola, Rev. Mod. Phys. 78, 217 (2006). 